\title{
Impaired Spatial Memory Performance in a Rat Model of Neuropathic Pain Is Associated with Reduced Hippocampus-Prefrontal Cortex Connectivity
}

\author{
Helder Cardoso-Cruz, ${ }^{1,2}$ Deolinda Lima, ${ }^{1,2}$ and Vasco Galhardo ${ }^{1,2}$ \\ ${ }^{1}$ Departamento de Biologia Experimental, Faculdade de Medicina do Porto, 4200-319 Porto, and 2Instituto de Biologia Molecular e Celular (IBMC), Grupo \\ de Morfofisiologia do Sistema Somatosensitivo, Universidade do Porto, 4200-319 Porto, Portugal
}

\begin{abstract}
Chronic pain patients commonly complain of working memory deficits, but the mechanisms and brain areas underlying this cognitive impairment remain elusive. The neuronal populations of the mPFC and dorsal CA1 (dCA1) are well known to form an interconnected neural circuit that is crucial for correct performance in spatial memory-dependent tasks. In this study, we investigated whether the functional connectivity between these two areas is affected by the onset of an animal model of peripheral neuropathic pain. To address this issue, we implanted two multichannel arrays of electrodes in the MPFC and dCA1 of rats and recorded the neuronal activity during a food-reinforced spatial working memory task in a reward-based alternate trajectory maze. Recordings were performed for 3 weeks, before and after the establishment of the spared nerve injury model of neuropathy. Our results show that the nerve lesion caused an impairment of working memory performance that is temporally associated with changes in the MPFC populational firing activity patterns when the animals navigated between decision points-when memory retention was most needed. Moreover, the activity of both recorded neuronal populations after the nerve injury increased their phase locking with respect to hippocampal theta rhythm. Finally, our data revealed that chronic pain reduces the overall amount of information flowing in the fronto-hippocampal circuit and induces the emergence of different oscillation patterns that are well correlated with the correct/incorrect performance of the animal on a trial-by-trial basis. The present results demonstrate that functional disturbances in the fronto-hippocampal connectivity are a relevant cause for pain-related working memory deficits.
\end{abstract}

\section{Introduction}

Evidence from both animal neurophysiological recordings and human brain imaging studies shows that the neural activity of medial prefrontal cortex (mPFC) and hippocampus correlates with the retention of information over a brief temporal scale, a function that is crucial for a wide range of cognitive tasks (Floresco et al., 1997; Jung et al., 1998; Rainer et al., 1999; Stern et al., 2001; Pesaran et al., 2002; Baeg et al., 2003; Schon et al., 2005). Important memory performance-related mPFChippocampus interactions occur via coherent oscillations in the theta frequency range (Hyman et al., 2005; Jones and Wilson, 2005b; Siapas et al., 2005; Lisman, 2010). These interactions were greatest just before a correct decision was made (Jones and Wilson, 2005a) and are evidence of a functional connection between both regions.

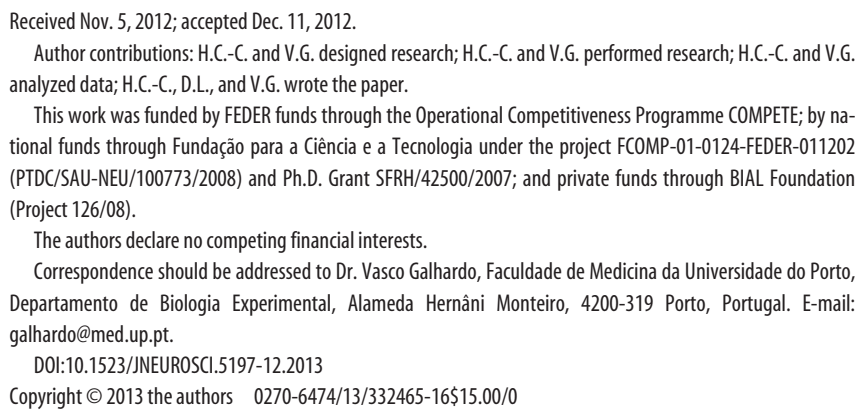

Impaired working memory is observed in several clinical conditions, such as traumatic brain injury (McDowell et al., 1997), mental retardation (Vallar and Papagno, 1993), schizophrenia (Green, 2006), attention deficit hyperactivity disorder (Frank et al., 1996), and chronic pain (Ling et al., 2007; Luerding et al., 2008). Indeed, it is commonly assumed that chronic pain may lead to learning dysfunctions (Kim et al., 2012). This clinical observation is corroborated by neuroimaging studies during noxious stimulation that demonstrated an activation of brain areas crucial for memory and learning (Peyron et al., 1998; Ploghaus et al., 2000; Ducreux et al., 2006; Schweinhardt et al., 2006; Buhle and Wager, 2010; Apkarian et al., 2011). Recent studies in animal pain models have also demonstrated that pain induces disturbances in different cognitive processes (for review, see Moriarty et al., 2011). Some of these pain-related cognitive impairments have been observed in spatial information acquisition (Cardoso-Cruz et al., 2011a), spatial memory (Cain et al., 1997; Lindner et al., 1999; Leite-Almeida et al., 2009; Hu et al., 2010), memory recognition (Cain et al., 1997; Lindner et al., 1999; Millecamps et al., 2004), emotional decision making (Pais-Vieira et al., 2009a, 2012; Ji et al., 2010), attentional performance (Millecamps et al., 2004; Boyette-Davis et al., 2008; PaisVieira et al., 2009b; Legrain et al., 2011), and aversive learning (Suzuki et al., 2007).

However, little is known about how pain affects the mPFChippocampal circuit. The $\mathrm{mPFC}$ region has an important role in pain modulation (Seifert et al., 2009; Devoize et al., 2011), and 
A

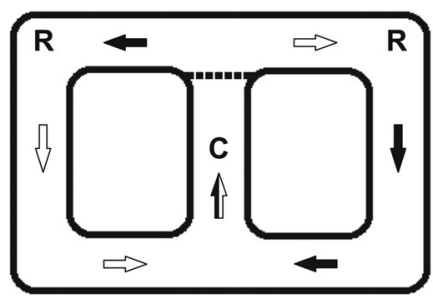

D
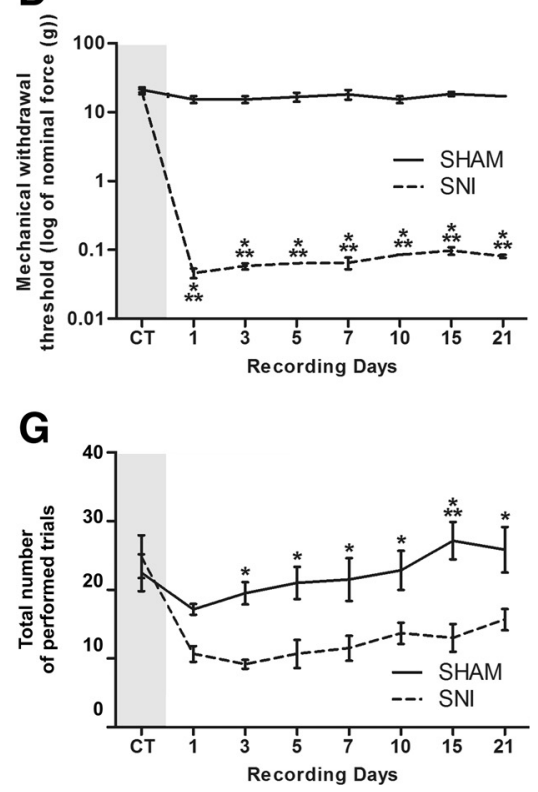

B

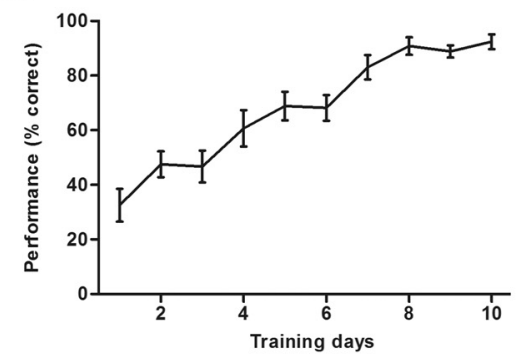

E

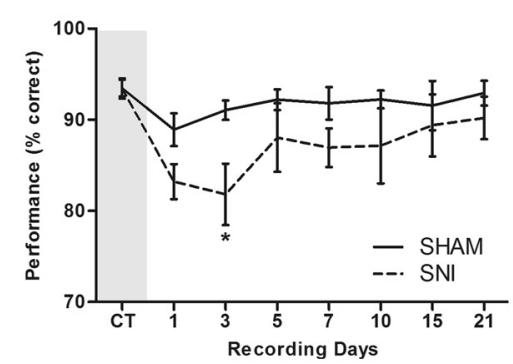

H

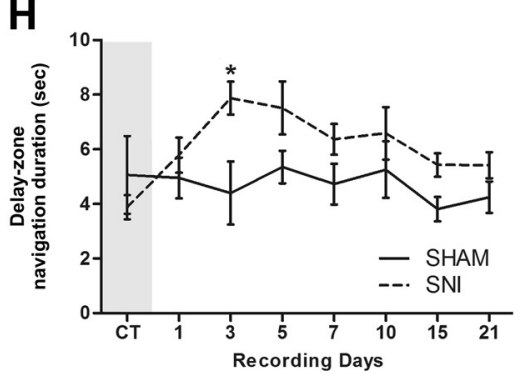

C
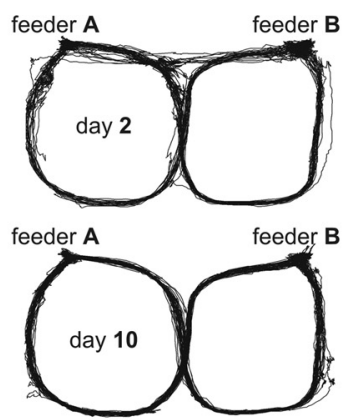

F

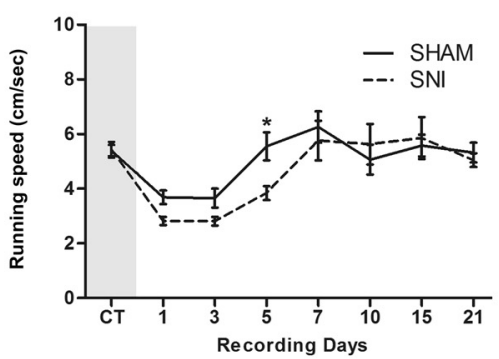

I

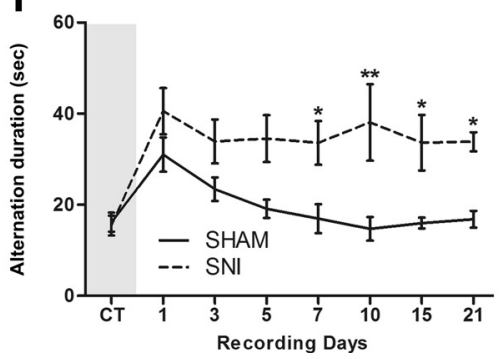

Figure 1. Apparatus and behavioral performance. $A$, Figure-eight maze, spatial alternation working memory task. Starting from the center of the maze (C), the animal had to alternately visit two reward sites (R) (feeder Aand B) to obtain chocolate-flavored pellets. The animal was required to come back to the center from a given reward site beforevisiting the other reward site. The arrows indicate the direction of travel when going to the left and right goals. $B$, Training period performance for the spatial working memory task. Only rats that reach at least the threshold of $80 \%$ of correct alternations from feeder A to feeder B according to the task imposed rules were selected to be candidates to receive the surgery for electrode implantation. C, Movement map of a rat across days 2 and 10 of the training period. As shown, the rat frequently made more navigation errors in the early days of training (see, for example, the direct trajectories between reward locations across day 2). $\boldsymbol{D}$, Level of sensibility to mechanical stimulation evaluated using von Frey filaments. A large decrease was observed in the threshold required to induce a paw response in the SNI group. $\boldsymbol{E}$, Recording period performance for the spatial working memory task. $\boldsymbol{F}$, A significant decrease in performance level and running velocity was observed in the SNI group after nervelesioning. G, Total number of trials performed in each recording session. A large decrease of the number of performed trials was observed in the $\mathrm{SNI}$ groups after nervelesioning. $\boldsymbol{H}$, The SN lanimals spent more time navigating in the delay zone of the behavioral test after lesioning. $\boldsymbol{I}$, Also a significant increase of the average interval between correct alternations was observed in this experimental group. Values are presented as the mean \pm SEM. SNI group, $n=5$; sham group, $n=5$. Comparisons between experimental groups are based on two-factor repeated-measures ANOVA, followed by post hoc Bonferroni test. ${ }^{*} p<0.05,{ }^{* *} p<0.01,{ }^{* * *} p<0.001$.

pain-induced plasticity of mPFC region may provide a cellular basis for cognitive impairments (Metz et al., 2009). Additionally, chronic pain has been associated with working memory deficits due to hippocampal LTP inhibition (Kodama et al., 2007; Ren et al., 2011), and abnormal cytokine expression (Al-Amin et al., 2011; del Rey et al., 2011), while reduction in hippocampal volume in chronic pain patients has been suggested to underlie learning and emotional deficits (Mutso et al., 2012). Despite all the knowledge gathered in recent years, it remains unclear how pain affects the share, maintenance, and processing of information that is crucial for spatial mnemonic processes. Therefore, our interest was to study how the mPFC-hippocampal connectivity is affected during a neuropathic condition.

\section{Materials and Methods}

\section{Animal model and ethical statement}

Fifty-one adult male Sprague Dawley rats weighing between 275 and $320 \mathrm{~g}$ were used in this study. The rats were maintained on a $12 \mathrm{~h}$ light/ dark cycle, and both training and recording sessions run at approximately the same time each day during the light portion of the cycle. All animals were food deprived to $90-95 \%$ of their ad libitum feeding body weights during the course of these experiments. All procedures and experiments adhered to the guidelines of the Committee for Research and Ethical Issues of the International Association for the Study of Pain (Zimmermann, 1983), with the Ethical Guidelines for Animal Experimentation of the European Community Directive Number 86/609/ECC of November 24, 1986, and were approved by national and local boards.

\section{Mazes and training procedures}

We used two distinct mazes in this study. Most experiments were based in a behavioral task consisting of a food-reinforced delayed spatial alternation task on a figure-eight-shaped maze, in a paradigm used in previous studies of episodic memory (Baeg et al., 2003; Ji and Wilson, 2007). The total dimension of the arena was $90 \times 60 \mathrm{~cm}$, with Plexiglas corridors $15 \mathrm{~cm}$ wide and opaque walls $30 \mathrm{~cm}$ high with intramaze cues in the reward locations (Fig. 1A). Starting from the center of the maze (Fig. $1 A$, C), the rats were trained to alternately visit two reward sites (Fig. $1 A, \mathrm{R}$ ) to obtain one chocolate-flavored pellet $(45 \mathrm{mg}$ ) that was automatically delivered by a pellet dispenser (Coulbourn Instruments). After visiting one of the reward locations, the animal had to continue forward and cross again the central corridor before visiting the opposite reward site; 
food rewards were not dispensed if the animal failed to cross the central corridor before arriving at the reward sites or if the animal made two consecutive visits to the same reward site (Fig. $1 \mathrm{~A}$, arrows illustrate the pattern of correct maze navigation). Control of pellet dispensers was fully automated using the OpenControl software adapted to this particular task (Aguiar et al., 2007). In all the analysis of this study, we have considered three different zones in the behavioral arena: the "reward zones" are the $15 \times 15 \mathrm{~cm}$ corner areas where the animal receives a pellet upon a correct alternation; the "delay zones" comprise the area between the reward zones and the central corridor; finally, the "choice zone" is the area preceding the reward zones and immediately following the central corridor (Fig. 1A). The testing room was moderately illuminated and rich in visual cues distant from the maze. During the training period (10 d), the rats were given three daily 10 min sessions to learn the alternation schedule. An error was defined by a consecutive visit to the same reward site or when the return route was the same as the approach route. Errors were not reinforced. Only animals that reached $80 \%$ of correct maze alternations during the training period were selected to continue the testing protocol of neuronal recordings (Fig. 1B).

The second maze used in a few experiments was a classical nonsample to match the T-maze, in which the animals need to alternate from the forced visited arm to receive a food reward. The arena consisted of a T-shaped arena with three sections that were $45 \mathrm{~cm}$ long and $15 \mathrm{~cm}$ wide, and opaque walls $30 \mathrm{~cm}$ high with intramaze cues in the reward locations; trials were initiated from the base of the T. Before alternation testing, the animals were habituated during $2 \mathrm{~d}$ to the T-maze with both food wells filled (four daily sessions of $3 \mathrm{~min}$ duration and $10 \mathrm{~min}$ intertrial intervals). The daily testing session was composed of 10 groups of two trialsone forced and one choice trial- both with an individual duration of 2 min. In the forced trial, only the baited sample arm is accessible. After the animal has retrieved the reward, the animal is placed back in the start area and both arms are made available for the choice trial. Correct arm entry results in reward, whereas incorrect arm entry results in absence of reward. An intertrial interval of 3 min was used between trials. Each session contained an equal number of left and right arm baited trials.

\section{Experimental protocols}

Experiment 1-spatial performance. The first experiment addressed the effect of the onset of the neuropathic pain model on working memory performance in the figure-eight-shaped spatial alternation task. A total of 22 animals were included in this experiment. One group of 10 animals ( $n=5$ in both sham and nerve-lesioned groups) was surgically implanted with two arrays of eight isonel-insulated tungsten filaments $(35 \mu \mathrm{m}$ in diameter) for the recording of extracellular single-unit and local field potentials (LFPs) while performing the spatial alternation task (see details below). After $7 \mathrm{~d}$ of recovery from the implantation surgery, the animals were retrained for $5 \mathrm{~d}$ to recover the presurgery performance level. Neuronal recordings were then done during 5 consecutive days [hereafter called the baseline or control period (CT)] while performing the maze alternation task in two daily sessions of 10 min with a $4 \mathrm{~h}$ interval between sessions. On the following day, the animals were briefly anesthetized and either surgically subjected to the spared nerve injury (SNI) model of neuropathic pain (Decosterd and Woolf, 2000) or to a sham intervention with the same extent of skin incision and muscle dissection. SNI surgery consists of ligation and transection of the tibial and common peroneal branches of the sciatic nerve, while sparing the sural nerve. Both interventions were done on the hindpaw contralateral to the side of implantation of the recording arrays. Behavioral and neural recordings in both groups were then resumed for a period of 3 weeks (with recordings performed at days 1, 3, 5, 7, 10, 15, and 21 after SNI or sham intervention). The sensory threshold for mechanical stimulation was assessed $1 \mathrm{~h}$ after the end of the second daily recording session by placing the animals in a cage with a metal mesh floor and touching the plantar surface of the paw with von Frey filaments (Somedic) as previously described (Chaplan et al., 1994; Cardoso-Cruz et al., 2011a).

Experiment 2-spatial learning performance in neuropathic animals. To study the effects of chronic pain on performance from a naive status, we evaluated the learning curves of groups of animals first exposed to both spatial alternation tasks $7 \mathrm{~d}$ after the SNI or sham intervention (eight- shaped arena: $n=6$ in sham group, $n=6$ in nerve-lesioned group; T-maze task: $n=6$ in sham group, $n=9$ in nerve-lesioned group). The learning performance of the treatment groups was followed for 10 consecutive days.

Experiment 3-hippocampal-function performance dependency. Additionally, we also validated the functional hippocampal dependency in both spatial alternation tasks. In this experiment, the animals were subjected to a training protocol of $10 \mathrm{~d}$ (as detailed above), and after that they received a hippocampal lesion or a sham intervention. Hippocampal lesions were made by bilateral injections of quinolinic acid (QA) (Sigma Chemical) centered at the dorsal CA1 region (dCA1) using the same described coordinates for multielectrode implantation $(0.4 \mu \mathrm{l}$ of a $0.18 \mathrm{M}$ solution, $\mathrm{pH}$ 7.4) (see details below). Control animals received bilateral injections of $0.4 \mu \mathrm{l}$ of artificial CSF at the same coordinates. ACSF contained the following (in $\mathrm{mM}$ ): $\mathrm{NaCl} 115.0, \mathrm{KCl} 5.6, \mathrm{CaCl}_{2} 2.0, \mathrm{MgCl}_{2} 1.0$, glucose 11.0, $\mathrm{NaH}_{2} \mathrm{PO}_{4}$ 1.0, and $\mathrm{NaHCO}_{3}$ 25.0, pH 7.4. Each rat was given $5 \mathrm{~d}$ to recover from surgery before testing. Postsurgery behavioral performance was evaluated during the following 6,8 , and $10 \mathrm{~d}$.

\section{Multielectrode implantation}

The procedure for the surgical implantation of intracranial multielectrode arrays has been described in detail previously (Cardoso-Cruz et al., 2011a). Briefly, animals were anesthetized with a ketamine/xylazine mixture $(10$ and $60 \mathrm{mg} / \mathrm{kg}$, respectively, i.m.). Anesthesia was maintained with small additional injections of ketamine (one-third of the initial dosage). Depth of anesthesia and paralysis of the musculature was assessed by regular testing of the corneal blink, hindpaw withdrawal, and tail-pinch reflexes. Core body temperature was maintained at $37^{\circ} \mathrm{C}$ through use of a homeothermic blanket system (Harvard Apparatus). Animals were secured in a stereotaxic frame using ear bars, and the skull was exposed and cleaned using hydrogen peroxide. Holes were bored in the skull to allow the implantation of four to five screws and the two microelectrode arrays. The screws were used for securing probes and for grounding purposes. Just before implantation, microelectrode array filaments were cut to the ideal length using sharp tungsten scissors and then were soaked in a saturated solution of sucrose. Each microelectrode array contained eight filaments of isonel-coated tungsten wire $(35 \mu \mathrm{m}$ in diameter) (California Fine Wire Company) with impedances varying between 0.5 and $0.7 \mathrm{M} \Omega$ at $1 \mathrm{kHz}$. The microelectrode arrays were assembled in $4 \times 2$ architecture, with spacing of $250 \mu \mathrm{m}$ between filaments and $450 \mu \mathrm{m}$ between each row. The arrays were mounted in the holder of a hydraulic micropositioner (FHC) and subsequently slowly driven $(50 \mu \mathrm{m} / \mathrm{min})$ to the $\mathrm{mPFC}$ and hippocampal dCA1 field. The following coordinates (in millimeters) relative to bregma (Paxinos and Watson, 1998) were used to place the arrays: $\operatorname{mPFC}(-2.2$ rostrocaudal, 1.0 mediolateral, 3.2 dorsoventral); and dCA1 (+3.2 rostrocaudal, 2.2 mediolateral, 2.7 dorsoventral).

\section{Neural recordings}

All neural activity signals from the implanted microelectrodes were recorded and processed using a Multineuron Acquisition Processor system (16-MAP, Plexon). Neural signals were preamplified (10,000-25,000×) and digitized at $40 \mathrm{kHz}$. Voltage time threshold windows were used to identify single-unit waveforms. Up to two neuronal action potentials per recording channel were sorted on-line (SortClient 2.6., Plexon) and were validated by off-line analysis (Offline Sorter 2.8., Plexon) according to the following cumulative criteria: voltage threshold $>2$ SDs of the amplitude distribution; signal-to-noise ratio $>3 ;<1 \%$ of interspike intervals $<1.2 \mathrm{~ms}$; and stability of the waveform shape as determined by a waveform matching template algorithm and principal component analysis. In addition, the Waveform Tracker software (Plexon) was used to verify that the recorded units were stable across experimental sessions. Extracellular LFPs were simultaneously recorded from the same implanted microwires by low-frequency $(0.5-200 \mathrm{~Hz})$ filtering of the raw signals. LFPs were preamplified and digitized at $500 \mathrm{~Hz}$. An overhead video tracking system (CinePlex 2, Plexon) was used to provide information about the animal position on the maze and to synchronize the video recordings with the acquired neuronal data. 


\section{Data analysis}

Neural data were processed off-line using NeuroExplorer 4 (NEX, Plexon) and exported to our own MatLab R14 (MathWorks) routines for additional analysis. The Kolmogorov-Smirnov (KS) test was used to determine whether datasets were normally distributed (Prism 5.0, GraphPad). Statistical comparisons of the experimental groups were performed by a two-factor ANOVA [repeated-measures (rmANOVA)], and when appropriate a post hoc analysis was performed using the Bonferroni test. The level of significance was set as $5 \%$. Results are expressed as the mean \pm SEM.

Behavioral data. Custom MatLab and Python scripts were used to sort behavioral intervals based on previous or next trial outcome (correct vs incorrect), maze navigation zone (reward, delay, choice zones), and by experimental session (control vs CFA). These measurements were calculated based on tracking navigation vectors obtained from behavior video recordings. Several parameters concerning the behavioral performance and navigation maps were examined across experimental sessions: percentage of correct alternations, total number of performed trials, averaged running speed, mean time of navigation across the delay zone, and average interval between two consecutive correct alternations per session.

Spiking activity. Average firing rate activity for the mPFC and dCA1 neurons was examined across maze navigation zones, comparing SNI and sham animals in presurgery and postsurgery recording sessions. To examine which neurons displayed elevated activity in the delay zone navigation, we compared for each alternation the average firing rate of the last second spent in the delay zone versus the global firing rate of the session (paired $t$ test, $p<0.05$ ).

Additionally, to analyze firing activity response for correct versus error alternations during each recording session, individual perievent time decision histograms were computed for each recorded neuron and plotted in a $2 \mathrm{~s}$ range centered at the time of the transition between the delay and the choice navigation zones ( $50 \mathrm{~ms}$ per bin). A KS test $(p<0.05)$ was used to test differences in the firing distribution of each neuron. The units were classified based in their increased, decreased, or unchanged firing rate. All recording sessions selected for comparisons of neuronal activity for correct versus error alternations had at least 5\% of incorrect alternations.

To characterize the temporal structure of the spiking activity in respect to the theta cycle, the raw hippocampal LFPs were filtered in the theta range (4-9 Hz) using a zero-phase forward and reverse digital four-pole Butterworth bandpass filter to yield the signal $\mathrm{LFP}_{\theta}$. The relationship between temporal structure of $\mathrm{MPFC}$ and $\mathrm{dCA} 1$ neuronal spiking activity and hippocampal ongoing theta rhythm was calculated using standard cross-correlograms (Neuroexplorer 4, Plexon). Correlations were computed using dCA1 $\mathrm{LFP}_{\theta}$ as reference with a temporal resolution of $2 \mathrm{~ms}$ per bin, and the rate histograms for both $\mathrm{mPFC}$ and CA1 spiking activity were calculated individually for each cell and are represented in the function of the populational activity across navigation zones. We compared the signals from the control period versus the signals from only one postsurgery session and selected day 10 because the nerve-lesioned animals reach stable levels of pain at this time point. The comparison of phase distributions was performed using a two-sample KS test $(p<0.05)$.

Spectral analysis. Global spectral characteristics of mPFC-dCA1 LFP signals were calculated for each navigation zone of the test arenas. The power spectral density (PSD) of mPFC $\left(P_{x x}\right)$ and CA1 $\left(P_{y y}\right)$ LFP signals was calculated between 1 and $50 \mathrm{~Hz}$ using the Welch method (MatLab native function), with 512-point fast Fourier transform of nonoverlapping $1 \mathrm{~s}$ epochs (Hanning window). Data are presented as the percentage of total PSD within the $1-50 \mathrm{~Hz}$ frequency range. Five frequency bands were considered: $1-4 \mathrm{~Hz}$ (delta), 4-9 Hz (theta), 9-15 Hz (alpha), 15-30 $\mathrm{Hz}$ (beta), and $30-50 \mathrm{~Hz}$ (gamma). To determine the spectral coupling among signals from the recorded regions, we calculated the correlation coefficient or coherence. Coherence $\left(\mathrm{C}_{x y}\right)$ was measured applying the equation mathematically equivalent to $\mathrm{C}_{x y}=\left|P_{x y}\right|^{2} /\left(P_{x} P_{y}\right)$, where the coherence for two signals, $x$ and $y$, is equal to the average cross-power spectrum $\left(P_{x y}\right)$ normalized by the averaged power spectra of the two signals. Its value lies between 0 and 1 , and it estimates for a given fre- quency the level to which phases are dispersed. $C_{x y}=0$ means that phases are dispersed, and high coherence $\left(C_{x y}=1\right)$ means phases of signals $x$ and $y$ are identical and the two signals are totally phase locked at this frequency.

The statistical method of partial directed coherence (PDC) was used to quantify the information flow within the mPFC-dCA1 circuit. The PDC method has been described in detail previously (Sameshima and Baccalá, 1999; Baccalá and Sameshima, 2001; Cardoso-Cruz et al., 2011b). Briefly, $\mathrm{PDC}$ is an alternative representation of multivariate processes involving Granger causality to uncover frequency domains of direct influence that allows for assessing interactions between brain regions and revealing their directionality. Its value lies between 0 and 1 PDC $=0$ can be interpreted as the absence of functional connectivity from the first structure $(j)$ to the second structure $(i)$ at a particular frequency window, while high PDC values indicate strong connectivity between the structures. This can be interpreted as the existence of information flow from brain structure $j$ to $i$.

\section{Histology}

After the end of all experiments, the rats were deeply anesthetized with ketamine/xylazine mixture and the recording site was marked by injecting DC current (10-20 $\mu \mathrm{A}$ for 10-20 s) through one microwire per array, marking the area below the electrode tips. After this step, the animals were perfused through the heart with $0.01 \mathrm{~m}$ phosphate buffer, $\mathrm{pH} 7.2$, in a $0.9 \%$ saline solution followed by $4 \%$ paraformaldehyde. Brains were removed, post-fixed in $4 \%$ paraformaldehyde for $4 \mathrm{~h}$, and stored in $30 \%$ sucrose before they were frozen and sectioned into $60 \mu \mathrm{m}$ slices. Sections were Nissl counterstained to help visualize the electrode tracks under the microscope.

\section{Results}

\section{Mechanical stimulation threshold}

All animals of the SNI group developed mechanical allodynia, as indicated by the significant decrease in the mechanical force needed to evoke paw withdrawal to von Frey filament stimulation in the hindpaw ipsilateral to the lesion (Fig. 1D). In the sham group, no statistical differences were noted in respect to the preoperation period (control period). A two-factor rmANOVA was used to compare responses from sham and SNI animals. Significant statistical differences were observed between experimental groups $\left(F_{(1,56)}=432.70, p<0.0001\right)$ and across recording days $\left(F_{(7,56)}=19.24, p<0.0001\right)$. Post hoc analysis revealed a significant decrease in the SNI after nerve lesioning (Bonferroni test, $p<0.001)$.

\section{Maze performance behavior}

Rats were trained to optimal performance on the figure-eight spatial alternation task before electrode implantation, and all animals performed the task at levels $>80 \%$ of correct choices after $10 \mathrm{~d}$ of training (three daily sessions of $10 \mathrm{~min}$ per animal) (Fig. $1 B)$. Figure $1 C$ shows two representative examples of cumulative animal trajectories in one session of day 2 and day 10 of the training period. As shown, the animal made more navigational errors in the early period of training (for example, see Fig. 1C, top, direct trajectories between the reward locations).

\section{Pain-related performance behavior}

To evaluate the chronic pain effects on the cognitive learning process, a set of animals already with pain were trained on a classic T-maze forced-choice task (Fig. 2A), and in the figureeight spatial delayed alternation task (Fig. $2 B$ ). In the T-maze task, there were significant differences between experimental groups $\left(F_{(1,130)}=73.26, p<0.0001\right.$; SNI group, $n=9$; sham control group, $n=6)$, and between daily training sessions $\left(F_{(9,130)}=3.95, p=0.0002\right)$. Post hoc analysis showed that SNI group performance levels were lower when compared with the 

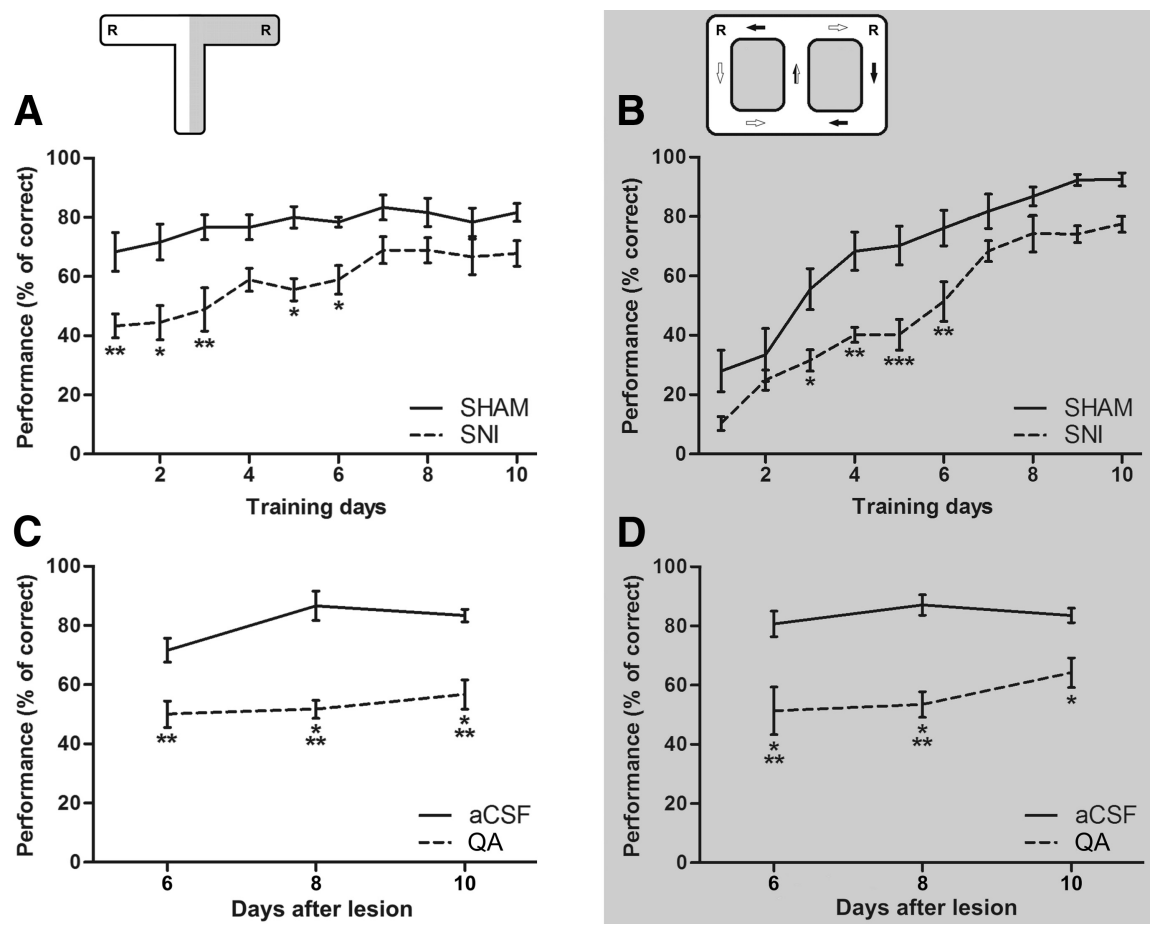

Figure 2. Pain-related performance behavior and hippocampus function performance dependency. $A, B$, The chronic pain effects on the cognitive learning process performance was tested on a classic T-maze forced-choice task $(\boldsymbol{A})$, and on the figure-eight spatial delayed alternation task ( $\boldsymbol{B}$ ). The nerve-lesioned animals (SNI:T-maze, $n=9$; figure-eightmaze, $n=6$ ) showed a significantly lower performance when compared with control animals (sham:T-maze, $n=6$; figure-eightmaze, $n=6$ ) across both tasks. A bilateral lesion of the dorsal CA1 hippocampal region was done with QA injection to evaluate whether the cognitive spatial performance depends on normal hippocampal function. $C, D, A$ clear decrease of the performance level was observed in hippocampus-lesioned group (QA group) across both behavioral tasks ( $\boldsymbol{C}$, T-maze: $Q A, n=6$; aCSF control, $n=6$; $\boldsymbol{D}$, figure-eight: $Q A, n=6$; aCSF-control, $n=6$ ). It should be noted that the performance decrease was larger in the figure-eight task. Values are presented as mean \pm SEM. Comparisons between experimental groups are based on two-factor repeated-measures ANOVA, followed by post hoc Bonferroni test. ${ }^{*} p<0.05$, ${ }^{* *} p<0.01$, ${ }^{* *} p<0.001$.

A

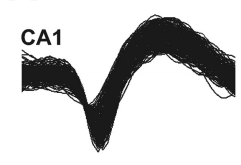

MPFC

D

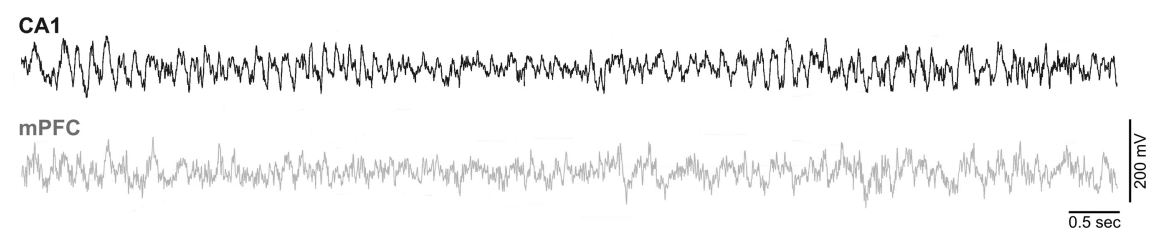

Figure 3. Stability of two cells simultaneously recorded in the same animal across experimental sessions and firing activity during task navigation. $\boldsymbol{A}$, Illustration of the waveform shape of a hippocampal CA1 cell (yellow) and an mPFC cell (green). Only units with a >3:1 signal-to-noise ratio were considered. $\boldsymbol{B}$, Off-line analysis of 3-D PC cluster stability from each channel shown across the whole recording sessions using the WaveTracker software (Plexon). C, In this view, 2-D PC clusters are projected as function of time (z-axis). Stability across time of the units isolated was used as an extra selection criteria. $\boldsymbol{D}$, Correspondent oscillations of intracranial LFP channels. Raw recordings represent 10 s of ongoing LFP activity.

sham group at day 1 (Bonferroni test, $p<0.01)$, day $2(p<0.05)$, day $3(p<0.01)$, day $5(p<0.05)$, and day $6(p<0.05)$ (Fig. $2 A)$. In the figure-eight spatial alternation task, ANOVA indicated significant differences between experimental groups $\left(F_{(1,100)}=\right.$
$70.81, p<0.0001$; SNI group, $n=6$; sham control group, $n=6)$ and between training sessions $\left(F_{(9,100)}=40.50, p<0.0001\right)$. In addition, post hoc analysis revealed a lower performance behavior of the SNI group when compared with the control group at day 3 (Bonferroni test, $p<0.05$ ), day $4(p<0.01)$, day $5(p<0.001)$, and day $6(p<0.01)$ (Fig. $2 B)$.

\section{Hippocampal function}

performance dependency

To evaluate whether the spatial cognitive performance depends on normal hippocampal function, we tested in both spatial alternation tasks a set of animals that received a bilateral lesion of the dCA1 hippocampal region. After the lesioning, a clear performance decrease was observed in both tasks (Fig. 2C,D). In the case of the T-maze task, ANOVA revealed statistical differences across experimental groups $\left(F_{(1,30)}=70.22, p<0.0001\right)$ as well as across testing sessions $\left(F_{(2,30)}=3.12, p<\right.$ 0.0580 ). In addition, post hoc analysis revealed that performance level was significantly lower in hippocampus-lesioned animals (QA group, $n=6$ ) when compared with control animals (ACSF group, $n=6$ ) at day 6 (Bonferroni test, $p<0.01$ ), day $8(p<0.001)$, and day $10(p<0.001)$ after hippocampal lesioning. In the case of the figure-eight spatial alternation task, ANOVA revealed differences between experimental groups $\left(F_{(1,30)}=46.79, p<\right.$ 0.0001; QA group, $n=6$; and ACSF control group, $n=6$ ), but no significant effect across testing sessions $\left(F_{(2,30)}=1.28\right.$, $p=0.2924)$. Post hoc analysis indicated that the performance in hippocampuslesioned animals decreased when compared with the control group at all testing sessions after surgical hippocampal lesioning [days 6 and 8 (with Bonferroni test), $p<0.001$; day 10 (with Bonferroni test), $p<0.05$ ].

\section{Spatial performance during recording} sessions

The performance of SNI rats $(n=5)$ was significantly worse than those in the sham group $(n=5)$ (Fig. 1E). ANOVA revealed statistical differences in the rat's performance between experimental groups $\left[F_{(1,56)}=12.97, p=0.0006\right.$; two-factor (groups $\times$ time) rmANOVA], and across recording days $\left(F_{(7,56)}=2.17, p=\right.$ 0.0482 ). Post hoc analysis revealed a significant performance decrease in the SNI group at days 3 and 5 after nerve lesioning (Bonferroni test, $p<0.05$ ). Additionally, a decrease in the running velocity was observed after lesioning in both experimental groups; both groups fully recovered to normal values of running speed $7 \mathrm{~d}$ after the surgery (Fig. $1 F$ ). ANOVA 
Table 1. Average of fronto-hippocampal populational firing rate activity when the animals ran the figure-eight spatial alternation task

\begin{tabular}{|c|c|c|c|c|c|c|c|c|}
\hline \multirow[b]{3}{*}{ Recording sessions (d) } & \multicolumn{4}{|l|}{$\mathrm{mPFC}$} & \multicolumn{4}{|l|}{ CA1 } \\
\hline & \multicolumn{2}{|l|}{ Sham } & \multicolumn{2}{|l|}{ SNI } & \multicolumn{2}{|l|}{ Sham } & \multicolumn{2}{|l|}{ SNI } \\
\hline & Mean & SEM & Mean & SEM & Mean & SEM & Mean & SEM \\
\hline CT & 4.18 & 0.24 & 4.78 & 0.42 & 3.68 & 0.19 & 3.78 & 0.12 \\
\hline 1 & 4.04 & 0.21 & 4.53 & 0.38 & 3.70 & 0.34 & 4.35 & 0.30 \\
\hline 3 & 4.22 & 0.09 & 4.62 & 0.43 & 3.83 & 0.35 & 4.88 & 0.35 \\
\hline 10 & 4.17 & 0.29 & 4.33 & 0.51 & 3.91 & 0.26 & 4.32 & 0.43 \\
\hline 15 & 4.16 & 0.26 & 3.96 & 0.53 & 3.63 & 0.25 & 3.95 & 0.32 \\
\hline 21 & 4.20 & 0.29 & 4.37 & 0.47 & 3.91 & 0.22 & 3.98 & 0.25 \\
\hline \multicolumn{9}{|l|}{ Delay zone } \\
\hline CT & 5.47 & 0.38 & 5.10 & 0.43 & 3.52 & 0.27 & 3.89 & 0.23 \\
\hline 1 & 5.50 & 0.29 & 5.04 & 0.33 & 3.36 & 0.30 & 4.02 & 0.35 \\
\hline 15 & 5.81 & 0.46 & 5.12 & 0.21 & 3.43 & 0.27 & 3.21 & 0.30 \\
\hline 21 & 6.04 & 0.50 & 5.30 & 0.16 & 3.45 & 0.24 & 3.36 & 0.27 \\
\hline \multicolumn{9}{|l|}{ Choice zone } \\
\hline CT & 3.46 & 0.17 & 4.00 & 0.38 & 3.82 & 0.14 & 3.56 & 0.12 \\
\hline 1 & 3.47 & 0.12 & 4.23 & 0.30 & 4.26 & 0.13 & 4.28 & 0.39 \\
\hline 3 & 3.51 & 0.18 & 4.01 & 0.24 & 4.02 & 0.17 & 5.52 & 0.64 \\
\hline 5 & 3.53 & 0.16 & 3.94 & 0.18 & 3.94 & 0.38 & 5.49 & 0.61 \\
\hline 7 & 3.66 & 0.15 & 3.98 & 0.42 & 4.07 & 0.33 & $5.85^{*}$ & 0.60 \\
\hline 10 & 3.57 & 0.15 & 4.17 & 0.28 & 3.84 & 0.15 & 5.03 & 0.46 \\
\hline 15 & 3.57 & 0.15 & 3.87 & 0.26 & 4.05 & 0.24 & 4.91 & 0.48 \\
\hline 21 & 3.53 & 0.17 & 3.70 & 0.23 & 3.73 & 0.50 & 4.89 & 0.53 \\
\hline
\end{tabular}

No significant differences were encountered in $\mathrm{mPFC}$ neurons; note, however, in this case that the average firing rate was significantly higher during the navigation in the delay zone when compared to reward and choice zones. In the case of $\mathrm{CA} 1$ an increase of the average firing rate was observed during reward and choice-zones navigation after nerve lesion. Values are presented as the mean \pm SEM Comparisons between CT and postsurgery recording sessions based on two-factor repeated-measures ANOVA, followed by post hoc Bonferroni test. ${ }^{*} p<0.05$.

showed significant differences between experimental groups $\left(F_{(1,56)}=2.34, p=0.0341\right)$ and a significant effect across recording days $\left(F_{(7,56)}=10.97, p<0.0001\right)$. In terms of the total number of trials performed by the experimental groups per recording session, a large decrease was observed in the SNI group after nerve lesioning when compared with control group (groups: $F_{(7,56)}=$ 20.21, $p=0.0012$; time: $F_{(7,56)}=6.31, p<0.0001$; Fig. $\left.1 G\right)$. Moreover, post hoc analyses revealed a significant decrease in performance of the SNI group across days 3, 5, 7, and 10 (Bonferroni test, $p<0.05$, respectively), day $15(p<0.001)$, and day $21(p<0.05)$.

An essential issue of alternation tasks is the temporal gap between trajectory decisions, in which the animal should maintain a retrospective memory of the previous visited reward location to choose correctly in the next left/right decision point. Our results show that after the nerve lesioning the SNI animals spent more time navigating in the delay zone of the behavioral test (groups: $F_{(1,56)}=12.15, p=0.0009$ ) (Fig. $1 H$ ). Post hoc analysis revealed an increase of the time of navigation in the delay zone for the SNI group at days 3 and 5 after lesioning (Bonferroni test, $p<0.001$ ). In addition, the interval of time that mediated two consecutive correct choices increased significantly for the SNI animals (Fig. 1I); ANOVA revealed a significant effect between experimental groups $\left(F_{(1,56)}=44.87, p<0.0001\right)$ and across the recording days after lesioning $\left(F_{(7,56)}=3.60, p=0.0025\right)$. Post hoc analysis revealed an increase of the interval between correct choices for SNI group after the nerve lesioning (Bonferroni test; days 5, 7, 15, and $21, p<0.05$; day $10, p<0.01)$.

\section{Neuronal activity}

In the present study, the Waveform Tracker Software (Plexon) was used to ensure that individual neural recordings were correctly identified across recording sessions (Fig. 3). Only neurons that remained stable throughout the recording sessions in terms of waveform shape and correct localization of the two recording sites confirmed by histological slices were considered in the analysis. A total of 175 validated neurons were recorded from the dorsal CA1 hippocampal region ( $n=85$ cells $)$ and $\mathrm{mPFC}$ region $(n=90$ cells $)$ in five nerve-injured rats (SNI; dCA1 $=43$ and $\mathrm{mPFC}=46$ cells $)$ and five control sham rats $(\mathrm{dCA} 1=42$ and mPFC $=44$ cells). Table 1 presents the average populational firing rate activity for the two recorded brain areas. No significant variations of neuronal activity were observed between animals during the presurgery period. The nerve injury caused an increase of the average firing rate of hippocampal dCA1 neurons when the animal was in the reward zone and choice zone, but not during navigation in the delay zone, when compared with the presurgery firing rates. In this case, we found a statistical effect in recording days [reward zone: $F_{(7,56)}=3.36, p=0.0046$; and choice zone: $F_{(7,56)}=4.06, p=0.0012$; two-factor (groups $\times$ time) rmANOVA]. Post hoc analysis revealed an increase of the average firing rate for SNI group at day 5 for reward zone $(p<0.05)$, and at day 7 for choice zone (Bonferroni test, $p<0.05$ ).

Spiking activity during the navigation on the delay zone

We calculated the percentage of neurons that elevated their average firing rate during the delay zone navigation in respect to the 


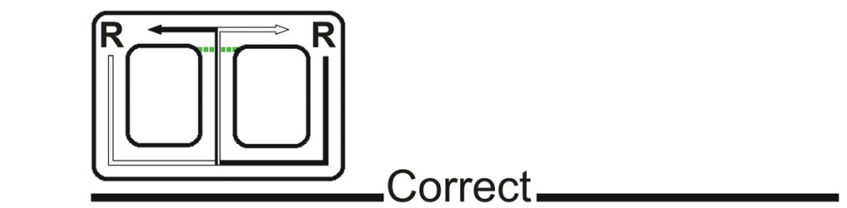

A
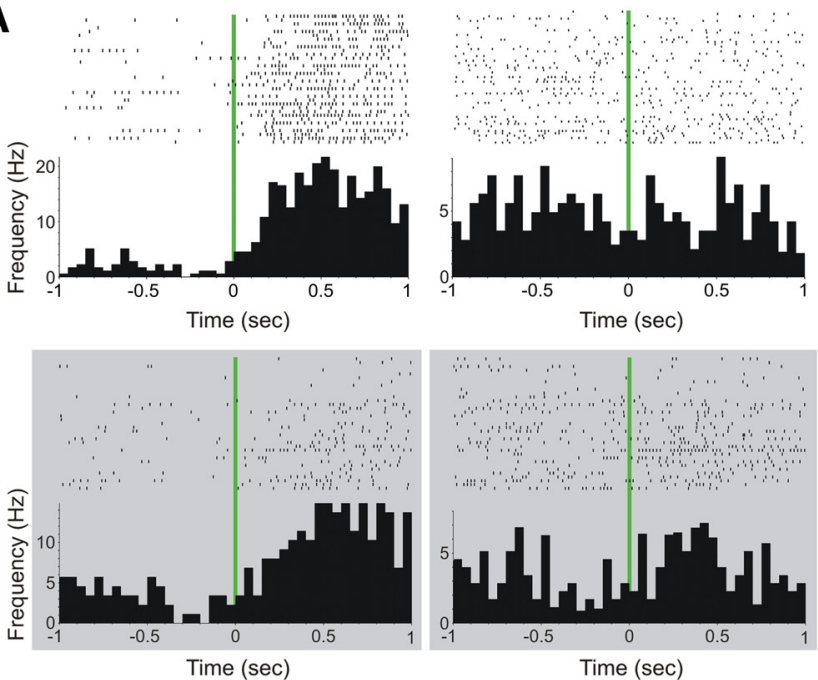

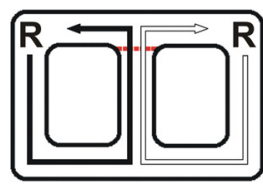

Error
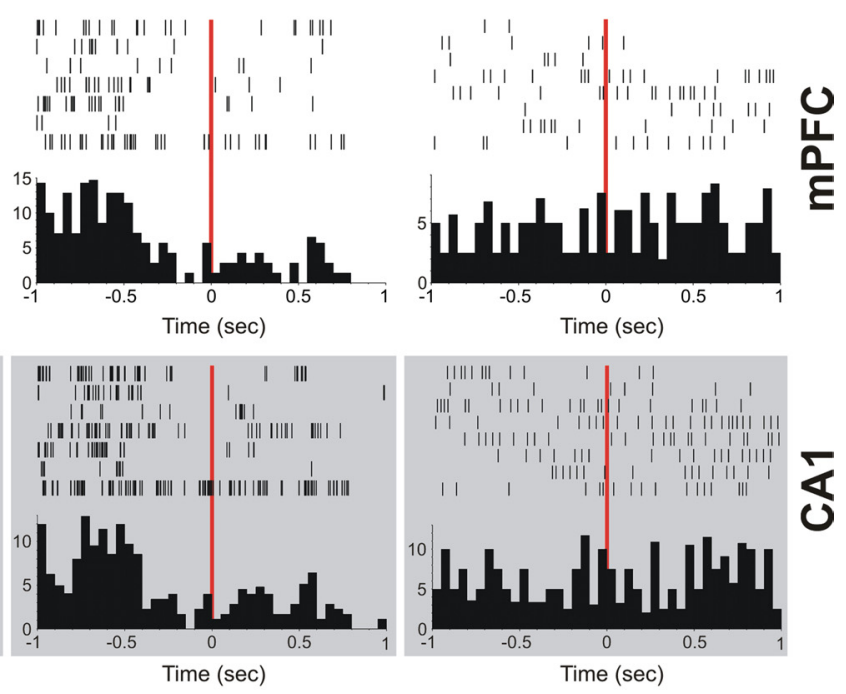
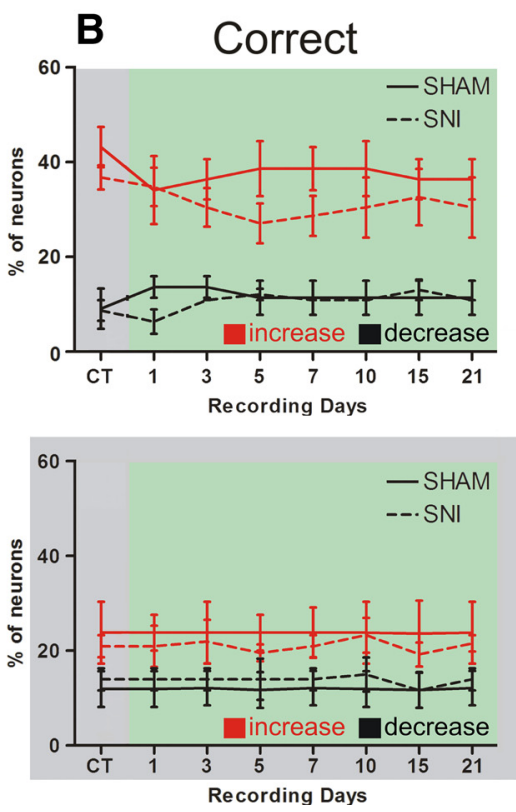
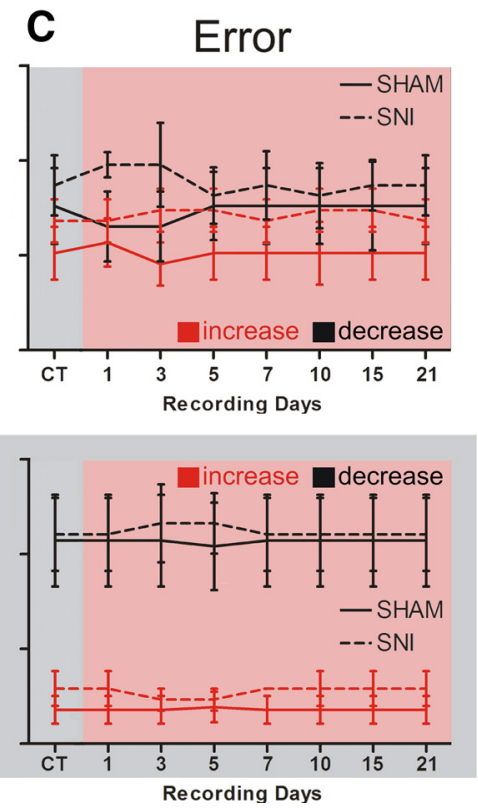
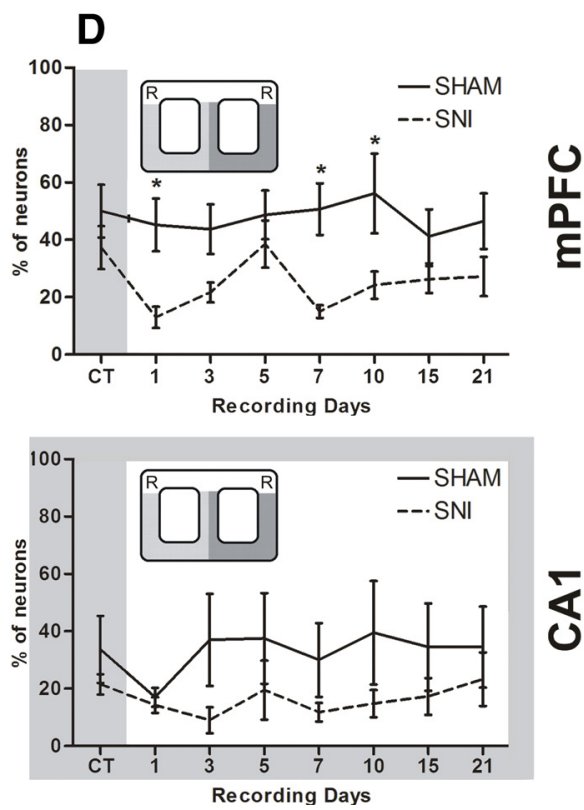

Figure 4. Typical neuronal responses for correct and error alternations. $A$, Perievent time decision histograms illustrate the averaged firing rate of four mPFC and four CA1 representative neurons around the decision limit (bin resolution of $50 \mathrm{~ms}$ ). Time $=0$ on the $x$-axis corresponded to the time of decision of direction turn to yield the reward location. Across both recorded regions, some neurons changed their spiking activity and others remain unchanged in respect to correct and error alternations after the decision of direction (from left to right, $\mathrm{mPFC}: \mathrm{KS}=0.32, p=0.0001 ; \mathrm{KS}=$ $0.07, p=0.8200 ; \mathrm{KS}=0.24, p=0.0282 ;$ and KS $=0.10, p=0.6725 ;$ from left to right $\mathrm{CA} 1 \mathrm{KS}=0.29, p=0.0003 ; \mathrm{KS}=0.15 p=0.1349 ; \mathrm{KS}=0.25, p=0.0026 ;$ and $\mathrm{KS}=0.13, p=0.4973$, respectively). $\boldsymbol{B}, \boldsymbol{C}$, The majority of $\mathrm{mPFC}$ neurons elevated their average firing activity when the rat performed a correct alternation $(\boldsymbol{B})$, and $C A 1$ neurons decreased their firing activity across error alternations ( $\boldsymbol{C}$. In both cases, no differences were found between experimental groups. $\boldsymbol{D}$, The percentage of neurons that elevated their average firing rate during the delay zone navigation with respect to the whole recording session decreased significantly after nerve lesioning in the case of mPFC neurons. Values are presented as mean \pm SEM. Comparisons between experimental groups are based on two-factor repeated-measures ANOVA, followed by Bonferroni post hoc test. ${ }^{*} p<0.05$.

whole recording session. Note that only the activity recorded in correct trials was selected for this analysis, so that direct comparisons could be made. From the pool of 175 neurons, we removed the neurons whose average firing rate in the delay zone was $<1$ $\mathrm{Hz}$ (mPFC-sham: 3 neurons; SNI: 6 neurons; dCA1-sham: 11 neurons; SNI: 9 neurons) because these provided too few spikes for a correct analysis (Floresco et al., 1997). In the case of mPFC neurons $(n=81)$, significant differences were found between experimental groups $\left[F_{(1,56)}=8.76, p=0.0182\right.$; two-factor (groups $\times$ time) rmANOVA], and no differences across recording days (Fig. 4D, top). Post hoc analysis revealed a decrease in the percentage of $\mathrm{mPFC}$ neurons that elevated their average firing rate within the delay zone at days 1,7 , and 10 after nerve lesioning (Bonferroni test, $p<0.05)$. In the case of dCA1 neurons $(n=65)$, no significant statistical differences were found between experimental groups and recording sessions (Fig. 4D, bottom). 
Firing activity of correct and error alternations

To analyze whether the firing activity was affected when the animal approached the decision point of left/right alternation, we calculated for each neuron the respective peridecision histogram of firing activity for either correct or error trials (Fig. 4A, green and red vertical lines, respectively). Figure $4 A$ presents representative examples of mPFC and dCA1 neurons with different activity patterns. The majority of the mPFC neurons increase their firing activity after the rat performed a correct direction choice (Fig. 4B, top). In contrast, the activity of dCA1 neurons commonly decreased across error alternations (Fig. 4C, bottom). More importantly, it should be noted that the analysis of firing patterns revealed no statistical differences in the percentage of neurons that increased their activity, decreased activity, or remained unchanged at the decision point between experimental groups (Fig. $4 B, C)$.

Changes in the hippocampal theta rhythm The relationship between the temporal structure of populational neuronal spiking activity and ongoing theta cycle of hippocampal LFPs was calculated for the presurgery period versus day 10 after sham or SNI surgery. As illustrated in Figure 5, different temporal activity patterns were observed for mPFC and dCA1 after peripheral nerve lesion surgery across the navigation zones of the arena. In the case of $\mathrm{mPFC}$, no differences were observed after nerve lesion surgery during the reward zone navigation between experimental groups. With respect to delay and choice zones, the mPFC neurons increased their firing precision after lesioning in relation to theta rhythm [delay zone: CT/SNI, $\mathrm{KS}=0.28, p=0.0459$; sham/SNI: $\mathrm{KS}=0.25, p=0.0257$; choice zone: $\mathrm{CT} / \mathrm{SNI}, \mathrm{KS}=0.26, p=0.0389$; sham/SNI: $\mathrm{KS}=0.26, p=$ 0.0291 (two-sample KS test)] (Fig. $5 A, B$ ). In the case of dCA1, no differences in the timing of spiking activity were observed between groups for reward and choice zones, while for the delay zone lesion the spiking activity was phase locked with respect to theta cycle after lesioning (CT/SNI: KS $=0.24, p=0.0289$; sham/ SNI: $\mathrm{KS}=0.26, p=0.0311$ ) (Fig. $5 C, D)$.

\section{Spectral analysis}

Changes in power spectral densities

A qualitative comparison of PSD values for $\mathrm{mPFC}$ and hippocampal dorsal CA1 LFPs is shown in Figure 6, comparing sham and SNI groups across recording sessions and maze navigation zones. The inspection of PSD confirmed that characteristic power oscillations were as expected, with high power in the theta frequency band $(4-9 \mathrm{~Hz})$ shared by both recorded areas, as has been previously described (Buzsáki, 2002; Lörincz et al., 2007). ANOVA revealed no statistical differences between experimental groups, but, as expected, a significant effect was found across
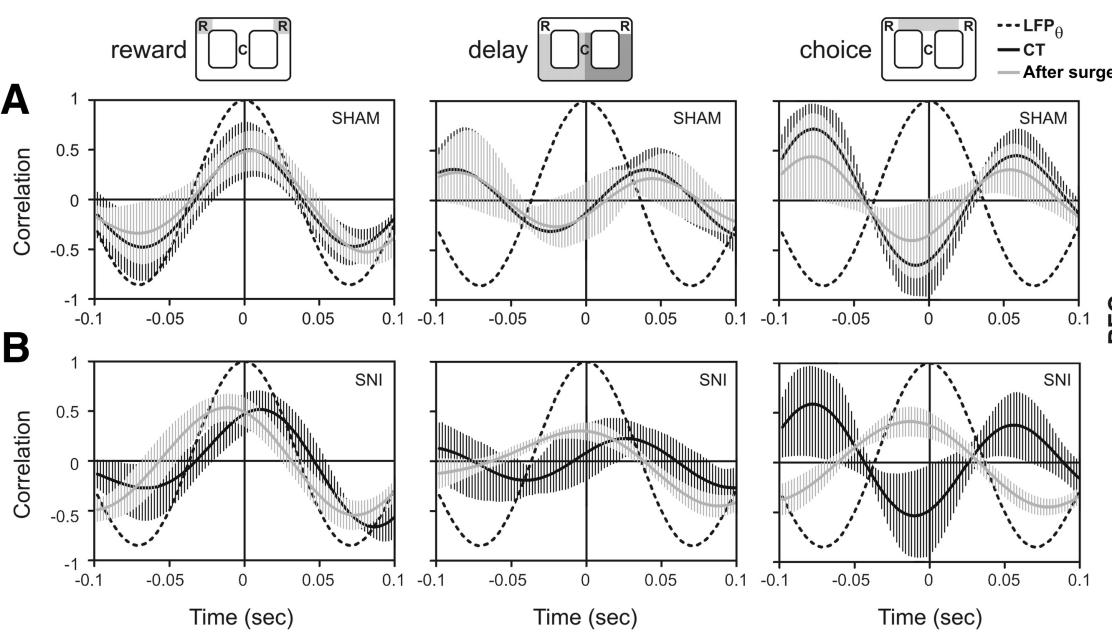

$\mathrm{NI}$
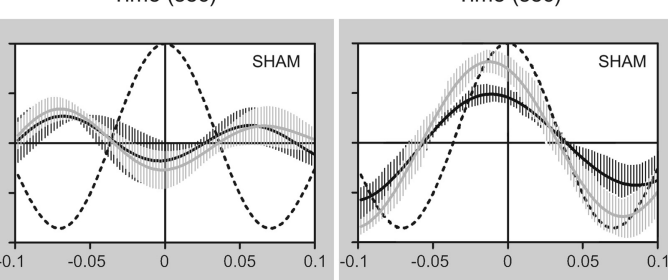

Figure 5. Correlation between hippocampal theta oscillations and spiking activity. The relationship between the temporal (the ongoing theta cycle of hippocampal LFPs (black dotted line), as calculated with respect to theta rhythms after nerve lesioning during the navigation across delay and choice zones (two-sample KolmogorovSmirnov test; delay zone: $\mathrm{CT} / \mathrm{SNI}, \mathrm{KS}=0.24, p=0.0459$; sham/SNI, KS $=0.25, p=0.0257$; choice zone: $\mathrm{CT} / \mathrm{SNI}, \mathrm{KS}=0.26, p=$ (O) choice zones, but across delay zone increased their firing precision (CT/SNI: KS $=0.24, p=0.0289$; and sham $/ \mathrm{SNI}: \mathrm{KS}=0.26, p=$ 0.0311). Values are mean \pm SEM.

frequency bands [mPFC: reward zone, $F_{(4,30)}=139.6, p<$ 0.0001 ; delay zone, $F_{(4,30)}=152.40, p<0.0001$; choice zone, $F_{(4,30)}=371.30, p<0.0001 ;$ dCA1: reward zone, $F_{(4,30)}=139.90$, $p<0.0001$; delay zone, $F_{(4,30)}=89.40, p<0.0001$; choice zone, $F_{(4,30)}=164.10, p<0.0001$; two-factor (groups $\times$ frequency bands) rmANOVA].

\section{Fronto-hippocampal coherence activity}

A qualitative comparison of the coherence activity measurements between the mPFC-dCA1 circuit LFPs across recording days and arena navigation zones is illustrated in Figure $7 A$. The global levels of the fronto-hippocampal coherence activity after peripheral nerve lesioning changed across recordings sessions (Fig. 7B), but not across frequency bands. In terms of statistical analysis, a significant effect was observed in the coherence activity with respect to recording sessions [reward zone: $F_{(7,56)}=26.89, p<0.0001$; delay zone: $F_{(7,56)}=15.78, p<0.0001$; choice zone: $F_{(7,56)}=10.62, p<0.0001$; two-factor (groups $\times$ time) rmANOVA]. Post hoc analysis revealed differences between experimental groups, in the reward zone at the first and last recording sessions (Bonferroni test, $p<$ 

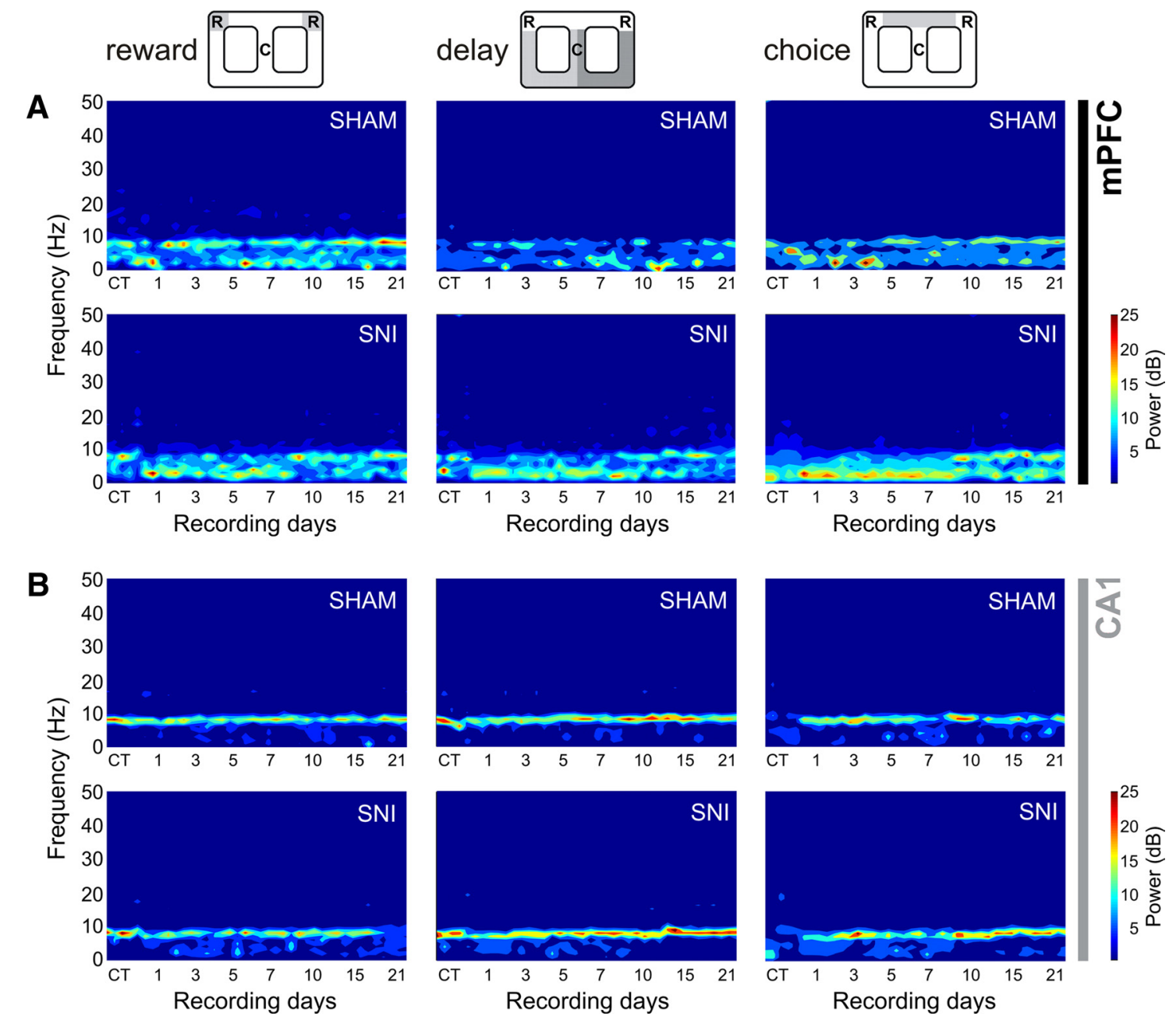

Figure 6. Spectral analysis of mPFC-dCA1 LFPs channels. $A, B, P S D$ of LFPs normalized by the percentage of total power within the frequency range analyzed (1-50 Hz) for mPFC $(\boldsymbol{A})$ and CA1 ( $B)$ channels, comparing the CT and sham or SNI recording sessions after surgery. Qualitative illustration of PSD showed that spectral power patterns were partially conserved across the experimental groups.

0.001; Fig. $7 B$, left); in the delay zone at the first and last two recording sessions $(p<0.05, p<0.01$, and $p<0.05$, respectively; Fig. $7 B$, middle); and in the choice zone at days $1(p<0.01)$ and $3(p<0.001)$ and the last recording session $(p<0.001)$ (Fig. $7 B$, right).

\section{Partial directed coherence}

The changes of mPFC-dCA1 circuit information flow in sham and SNI animals were determined by PDC analysis. Figure 8 illustrates the PDC activity across recording days and arena navigation zones. In the case of the direction from dCA1 to mPFC (Fig. 8A), the qualitative analysis confirmed strong PDC values at the theta frequency band $(4-9 \mathrm{~Hz})$ in the choice and also in the delay navigation zones, which is lost after peripheral nerve lesioning. In the opposite direction — from mPFC to dCA1 - high PDC values at the theta band are also visible in the choice zone, and they are also lost after the nerve injury (Fig. $8 B$ ).

Detailed analysis of averaged PDC values across the $1-50 \mathrm{~Hz}$ frequencies for each recording session showed that the levels of information flow decreased significantly after nerve lesioning (Fig. 9). The global PDC level is particularly decreased in the reward zone, in both circuit directions, and in the choice zone in the $\mathrm{dCA} 1 \rightarrow$ mPFC direction of information. In the case of dCA1 $\rightarrow$ mPFC direction, ANOVA for reward location revealed signif- icant differences between experimental groups $\left[F_{(1,56)}=21.17\right.$, $p=0.0066$; two-factor (groups $\times$ time) rmANOVA], and no effects across recording days. Post hoc analysis revealed a significant decrease of PDC level for SNI group at days 1, 7, and 15 after lesioning (Bonferroni test, $p<0.01$; Fig. 9A, left). For the delay zone, no differences were found between experimental groups, but a significant effect was found across recording sessions $\left(F_{(7,56)}=\right.$ 4.57, $p=0.0004)$. Post hoc analysis revealed a significant decrease of the PDC levels in the SNI group at day $15(p<0.001$; Fig. $9 A$, middle). With respect to choice zone, statistical differences were found between groups $\left(F_{(1,56)}=197.60, p<0.0001\right)$ as well as across recording sessions $\left(F_{(7,56)}=8.50, p<0.0001\right)$. Post hoc analysis revealed a large decrease in the PDC level for SNI group $(p<0.001$; Fig. 9A, right). In the case of the direction from $\mathrm{mPFC}$ to dCA1, a significant effect was observed between experimental groups when the animal was in the reward zone $\left(F_{(1,56)}=113.40\right.$, $p<0.0001)$, but no effect across recording sessions. Post hoc analysis revealed that after the lesioning the PDC level decreased significantly in the SNI group (days 1,3 , and 7, $p<0.05$; days 5 and $21, p<0.01$ ); days 10 and $15, p<0.001$; Fig. $9 C$, left). In the delay zone, ANOVA revealed no differences between groups, but there was a significant effect across time $\left(F_{(7,56)}=3.76, p=\right.$ 0.0021; Fig. $9 C$, middle). With respect to choice zone, no signif- 

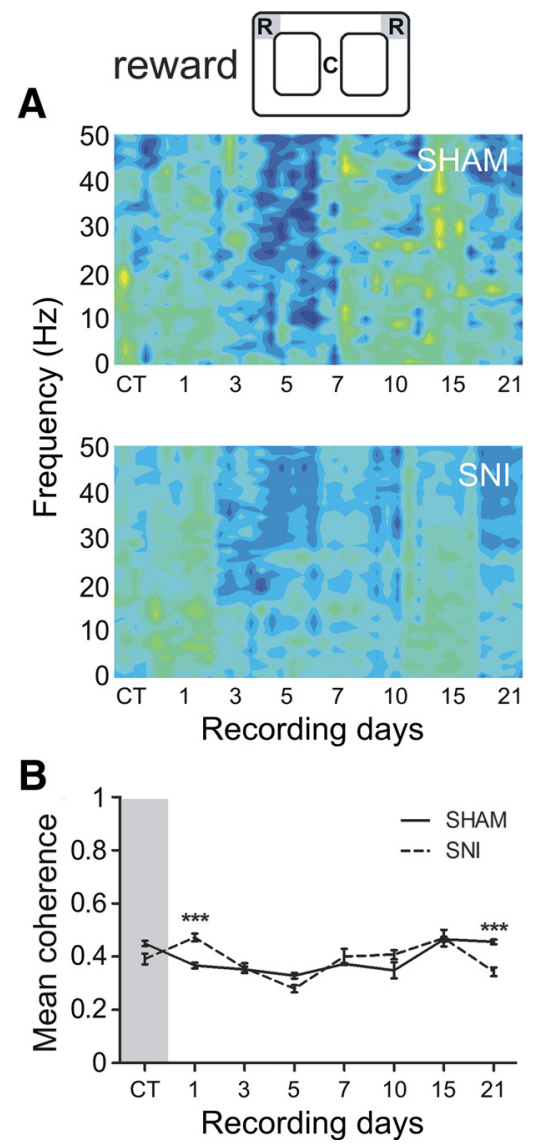
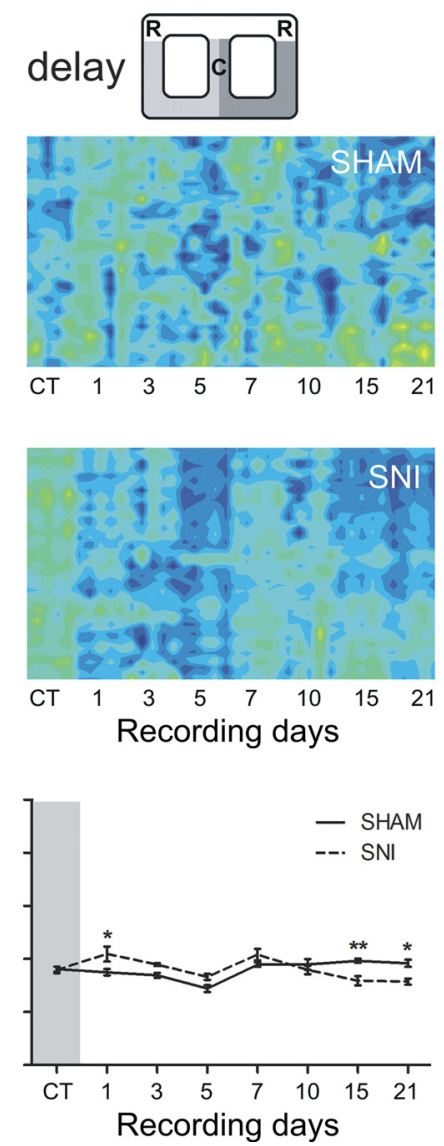
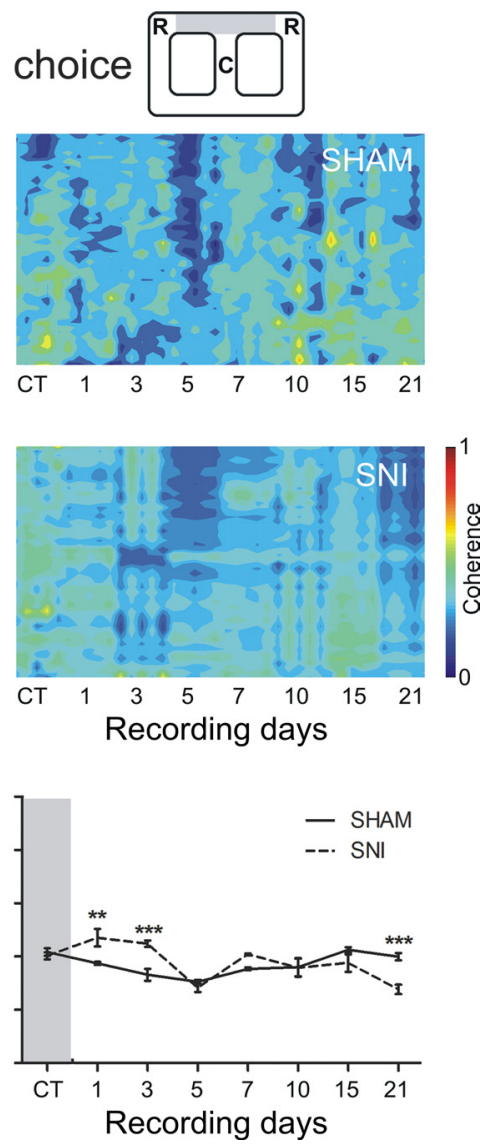

Figure 7. Spectral coherence. $\boldsymbol{A}$, Spectral coherence between simultaneously mPFC and CA1 LFPs recorded channels showed similar global levels of coherence activity across navigation zones for experimental groups. $\boldsymbol{B}$, The averaged coherence (in $1-50 \mathrm{~Hz}$ frequency range) across recording sessions indicated no significant differences between experimental groups; however, significant differences were found across recording sessions, particularly during the early recording sessions after lesioning. Values are presented as mean \pm SEM. SNI group, $n=5 ;$ sham group, $n=5$. Comparisons between experimental groups are based on two-factor repeated-measures ANOVA, followed by post hoc Bonferroni test. ${ }^{*} p<0.05,{ }^{* *} p<0.01,{ }^{* * *} p<0.001$.

icant differences were registered between experimental groups, but a significant effect was observed across recording sessions $\left(F_{(7,56)}=3.76, p=0.0021\right)$. Post hoc analysis revealed that at day 3 after lesioning the PDC was significantly lower in the SNI group compared with the sham group ( $p<0.05$; Fig. $9 C$, right).

To detail the fronto-hippocampal circuit information flow across frequency bands, we calculated the averaged PDC per range of frequencies (Fig. 9B). A two-way (groups $\times$ frequency bands) rmANOVA was performed to assess the differences in the averaged PDC between the sham and SNI groups. In the case of the $\mathrm{dCA} 1 \rightarrow$ mPFC direction, there was a significant difference between experimental groups (reward zone: $F_{(1,30)}=87.34, p<$ 0.0001 ; delay zone: $F_{(1,30)}=20.08, p<0.0001$; choice zone: $\left.F_{(1,30)}=326.89, p<0.0001\right)$, as well between frequency bands (reward zone: $F_{(4,30)}=13.16, p<0.0001$; delay zone: $F_{(4,30)}=$ $21.10, p<0.0001$; choice zone: $\left.F_{(4,30)}=39.86, p<0.0001\right)$. Post hoc analysis revealed that after the nerve lesioning the PDC level decreased at all frequency bands in the choice zone and in the reward zone, with the exception of delta band in the reward zone (Bonferroni test, $p<0.001$ ). In the delay zone, we found only a significant decrease at the theta frequency band $(p<0.001)$. In the case of the $\mathrm{mPFC} \rightarrow \mathrm{dCA} 1$ direction, there was a significant difference between experimental groups at the reward and choice zones (reward zone: $F_{(1,30)}=363.50, p<0.0001$; choice zone: $F_{(1,30)}=26.61, p<0.0001$ ), and between frequency bands (reward zone: $F_{(4,30)}=8.59, p<0.0001$; delay zone: $F_{(4,30)}=4.32$, $p=0.0039$; and choice zone: $F_{(4,30)}=81.91, p<0.0001$; Fig. $\left.9 D\right)$.
Post hoc analyses revealed that after the nerve lesioning the PDC level decreased in all frequency bands at the reward zone $(p<$ 0.001 ), and decreased in theta and alpha bands at the choice zone. No significant differences were found for the delay zone.

Information flow activity in correct and error alternations

We expanded the analysis of the lesion-induced changes in decision-related neuronal activity (Fig. $4 A$ ) by comparing the PDC values of the local field potential signals for the same periods of transition between the delay and the choice navigation zones, and again separating these transition periods into correct or incorrect alternations. Different patterns of PDC activity were observed across correct and error alternations for both directions of the circuit (Fig. 10A).

The quantitative analysis of the averaged PDC in the $1-50 \mathrm{~Hz}$ frequency range showed that the global levels of information flow decreased significantly after nerve lesioning (Fig. 10B). These changes were observed for both directions of the mPFC-dCA1 circuit in both correct and error alternations. In the case of dCA1 $\rightarrow$ mPFC direction, for correct alternations statistical differences were found for experimental groups $\left[F_{(1,56)}=17.77, p=0.0029\right.$; two-factor (groups $\times$ time) rmANOVA], but not across recording sessions. Post hoc analysis revealed a significant increase of the PDC level across day 21 after SNI lesioning (Bonferroni test, $p<$ $0.01)$. For error alternations, significant effects were observed across groups $\left(F_{(1,56)}=57.34, p<0.0001\right)$ and recording sessions $\left(F_{(7,56)}=4.35, p=0.0007\right)$. Post hoc analysis revealed a significant 

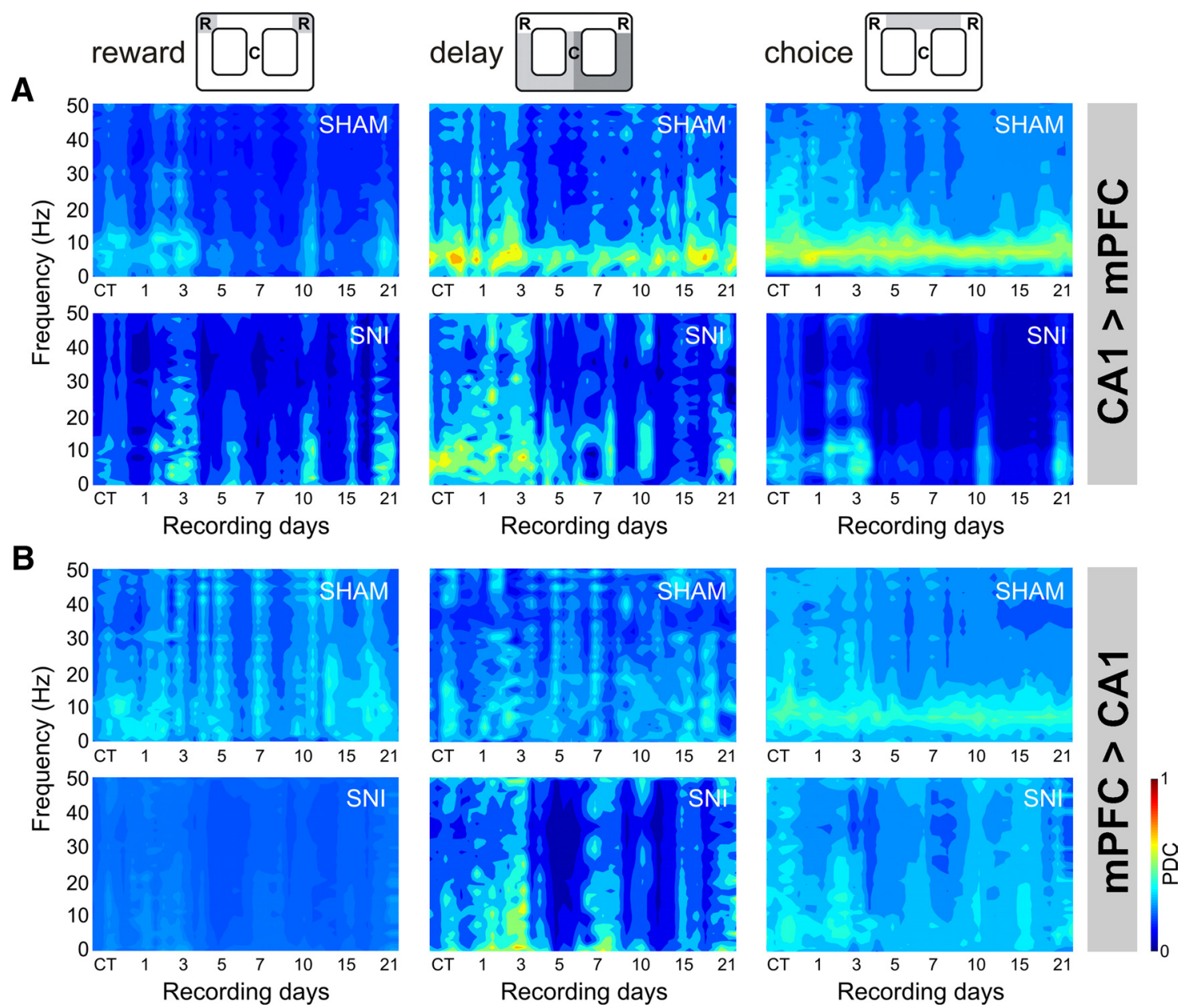

Figure 8. The oscillations of information flow between the two recorded regions were determined by partial directed coherence analysis. Data were presented individually across the three considered navigation zones of the behavioral task. $A, B$, The amount of information flow from dorsal hippocampal CA1 to $\mathrm{mPFC}$ after peripheral nerve lesioning decreased dramatically across choice zone navigation $(\boldsymbol{A})$, and from $\mathrm{mPFC}$ to $C A 1$ across the reward zone $(\boldsymbol{B})$, indicating that less information was processed in the $\mathrm{mPFC}-\mathrm{dCA} 1$ circuit after peripheral nerve lesioning.

decrease of the PDC activity across days 1 and 5 after nerve lesioning (Bonferroni test, $p<0.05$ ). In the case of $\mathrm{mPFC} \rightarrow \mathrm{dCA} 1$ direction, for correct alternations statistical differences were found for experimental groups $\left(F_{(1,56)}=21.74, p=0.0016\right)$, but not across recording sessions. Post hoc analysis revealed a significant decrease of the PDC activity across days 1 and 21 after SNI lesioning (Bonferroni test, $p<0.05$ and $p<0.01$, respectively). For error alternations, statistical differences were observed across groups $\left(F_{(1,56)}=60.86, p<0.0001\right)$, and recording sessions $\left(F_{(7,56)}=4.47, p=0.0005\right)$. Post hoc analysis revealed a significant decrease of the PDC level across days 1, 3, 5, and 21 after nerve lesioning (Bonferroni test, $p<0.001, p<0.001, p<0.05$, and $p<0.001$, respectively).

In relation to the frequency bands (Fig. 10C), the global PDC level was particularly decreased across theta, alpha, and delta bands for correct and error alternations. One exception was found across correct alternations, for the dCA $1 \rightarrow$ mPFC direction, which decreased across theta and increased for gamma frequency band after nerve lesioning. For the dCA1 $\rightarrow$ mPFC direction during correct alternations, we found significant differences between experimental groups $\left[F_{(1,30)}=4.83, p=0.0319\right.$; two-factor (groups $\times$ frequency bands) rmANOVA], and frequency bands $\left(F_{(4,30)}=41.96, p<0.0001\right)$. Post hoc analysis revealed a significant decrease of PDC activity after SNI lesioning in the theta frequency band (Bonferroni test, $p<0.01$ ), and an increase in the gamma frequency band $(p<0.01)$. On the other hand, for error alternations, statistical differences were found between groups $\left(F_{(1,30)}=30.42, p<0.0001\right)$ and frequency bands $\left(F_{(4,30)}=11.49, p<0.0001\right)$. Post hoc analysis revealed a significant decrease of the PDC level for theta (Bonferroni test, $p<0.01)$, alpha $(p<0.01)$, and beta $(p<0.05)$ bands after nerve lesioning. In the case of $\mathrm{mPFC} \rightarrow \mathrm{dCA} 1$ direction, for correct alternations significant differences were found between experimental groups $\left(F_{(1,30)}=41.38, p<0.0001\right)$, and across frequency bands $\left(F_{(4,30)}=18.14, p<0.0001\right)$. Post hoc analysis revealed a significant decrease of the PDC activity for the SNI group across theta (Bonferroni test, $p<0.001$ ), alpha $(p<0.01)$, and beta $(p<0.05)$ bands. For error alternations, a significant effect was found across experimental groups $\left(F_{(1,30)}=59.87, p<0.0001\right)$ and frequency bands $\left(F_{(4,30)}=9.31, p<0.0001\right)$. Post hoc analysis revealed a significant decrease of the information flow across theta (Bonferroni test, $p<0.001)$, alpha $(p<0.001)$, and beta $(p<0.05)$ bands for the SNI group.

\section{Discussion}

In this study, we report how the induction of chronic neuropathic pain affects the fronto-hippocampal functional connectivity by examining the temporal structure in spiking and LFP activity while rats performed spatial alternation tasks. The behavioral tests used in the present study were classical spatial working 
memory tasks, where the animals need to remember the route previously used to correctly alternate between reward sites.

We report that the pain-inducing SNI lesion impaired the spatial memory performance in these animals. These results are in accordance with recent studies using animal models of neuropathic pain that have also showed a reduction in spatial memory (Hu et al., 2010; Ren et al., 2011), and with clinical reports that chronic pain patients commonly present working memory impairments (Ling et al., 2007; Luerding et al., 2008). Similar memory impairments have been reported following prefrontal (Kyd and Bilkey, 2003) or hippocampal lesioning (Gaskin et al., 2009a,b), and during the inhibition of the mPFC-hippocampus circuit by lidocaine (Floresco et al., 1997) or muscimol infusions (Wang and Cai, 2006; Yoon et al., 2008).

Previous reports have shown an increase in the rate of mPFC neuron firing activity during the delay period in delayed-choice tasks (Miller et al., 1996; Fuster, 1997; Rainer et al., 1999), and this led to the suggestion that working memory processing may be dependent on increased activity of mPFC neurons. Our results show that the nerve lesion reduced the number of mPFC neurons with increased firing rates in the delay zone versus the other zones of the maze. This decrease in mPFC activity during the delay period is in agreement with the suggestion that mPFC instability may play a crucial role in pain-induced memory dysfunction (Metz et al., 2009; Apkarian et al., 2011). In contrast, the nerve lesion marginally affected the overall neuronal firing rate of the CA1 in which we observed an increase in the populational activity during navigation in the reward and choice zones. In addition, our data showed that the majority of the mPFC neurons increased their firing activity in the decision point when the rat performed a correct alternation, while the activity of dCA1 neurons decreased during error alternations. More importantly, these firing patterns remain unchanged after peripheral nerve lesioning.

Recordings from awake animals have shown that hippocampal neurons fire phase locked to the theta oscillation of the hippocampal field potential (Fox and Ranck, 1981; Skaggs et al., 1996), in which the supramammillary nucleus has an important role in its generation (Ruan et al., 2011). The robust hippocampal theta frequency band has also been reported to be responsible for theta phase-locked firing patterns of mPFC neurons (Hyman et al., 2005; Jones and Wilson, 2005a; Siapas et al., 2005; Sirota et al., 2008), and these phase interactions were greatest when the animal made a decision (Jones and Wilson, 2005a,b). Hyman et al. (2010), using a two-level delayed nonmatch-to-sample task reported that during error trials the $\mathrm{mPFC}$ neurons loose the theta
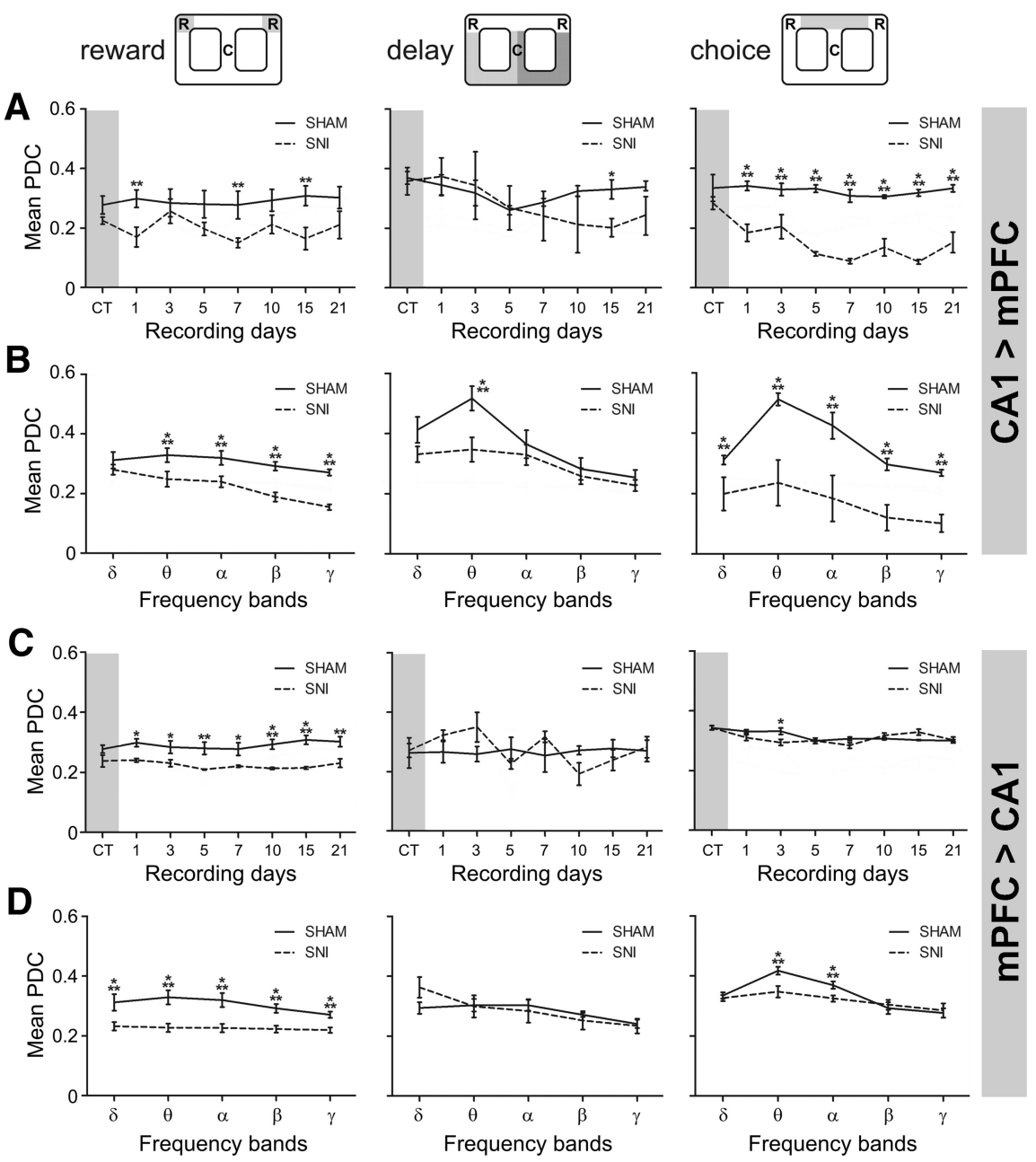

Figure 9. Partial directed coherence activity across recording days and frequency bands. $\boldsymbol{A}, \boldsymbol{B}$, The averaged PDC level in the 1-50 Hz frequency range across recording sessions indicates that less information was transmitted from CA1 to mPFC after frection ly the theta frequency showed a decreased after lesioning. $C, D$, In the $\mathrm{mPFC} \rightarrow$ CA1 direction, the averaged PDC level decreased zone across recording days $(\boldsymbol{C})$, and across frequency bands for reward (in all frequency bands) and choice (in theta and corresponds to the averaged PDC activity across postsurgery sham/SNI recording sessions. Frequency bands: delta $(\delta ; 1-4 \mathrm{~Hz})$, theta $(\theta ; 4-9 \mathrm{~Hz})$, alpha $(\alpha ; 9-15 \mathrm{~Hz})$, beta $(\beta ; 15-30 \mathrm{~Hz})$, and slow-gamma $(\gamma ; 30-50 \mathrm{~Hz})$. Values are presented as the mean \pm SEM. SNI group, $n=5$; sham group, $n=5$. Comparisons between experimental groups are based on two-factor repeatedmeasures ANOVA, followed by post hoc Bonferroni test. ${ }^{*} p<0.05$, ${ }^{* *} p<0.01,{ }^{* * *} p<0.001$.

entrainment that occurs via theta range interactions between $\mathrm{mPFC}$ and hippocampus, which is in agreement with human studies showing strong $\mathrm{mPFC}$-hippocampus theta coherence during working memory tasks (Tesche and Karhu, 2000; Onton et al., 2005). In fact, half of the mPFC neuronal population recorded by Hyman et al. (2010) presented theta entrainment across the correct trials, while only a limited number of mPFC neurons presented theta entrainment for the error trials. Moreover, we found that almost all of the MPFC neurons had a similar pattern of activity across correct and error trials.

Despite the differences between both behavioral tasks, we found that after nerve lesioning the spiking oscillatory activity of $\mathrm{mPFC}$ and dCA1 neurons increased their level of correlation with respect to the hippocampal theta rhythm, particularly during delay zone navigation. More importantly, it should be noted that we observed an increase of theta entrainment in the SNI group that was correlated with poor working memory performance; this is in 
A

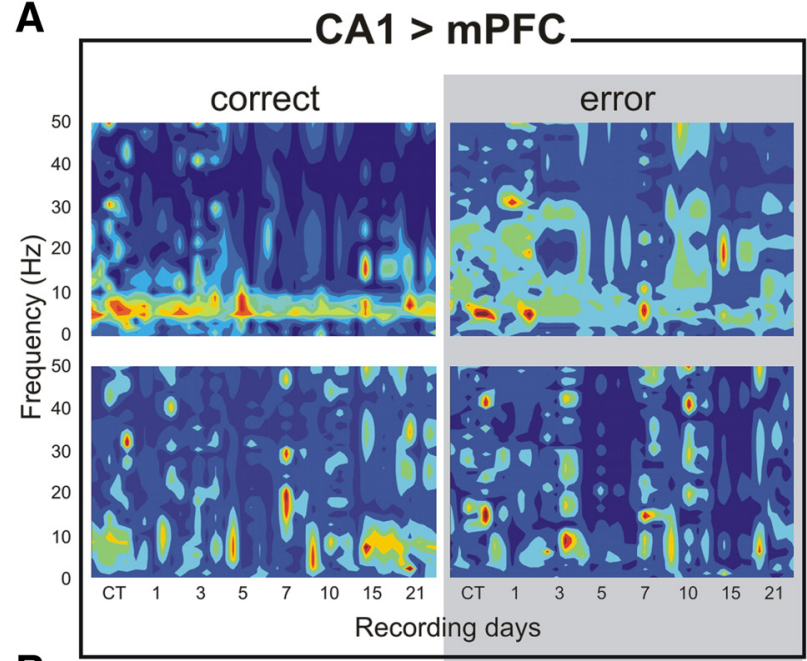

B
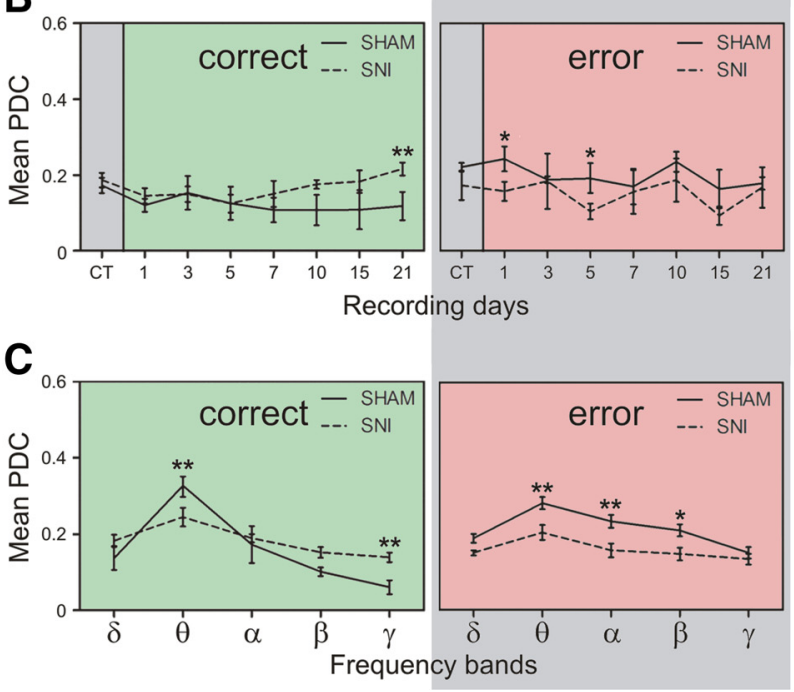

$\mathrm{mPFC}>\mathrm{CA} 1$
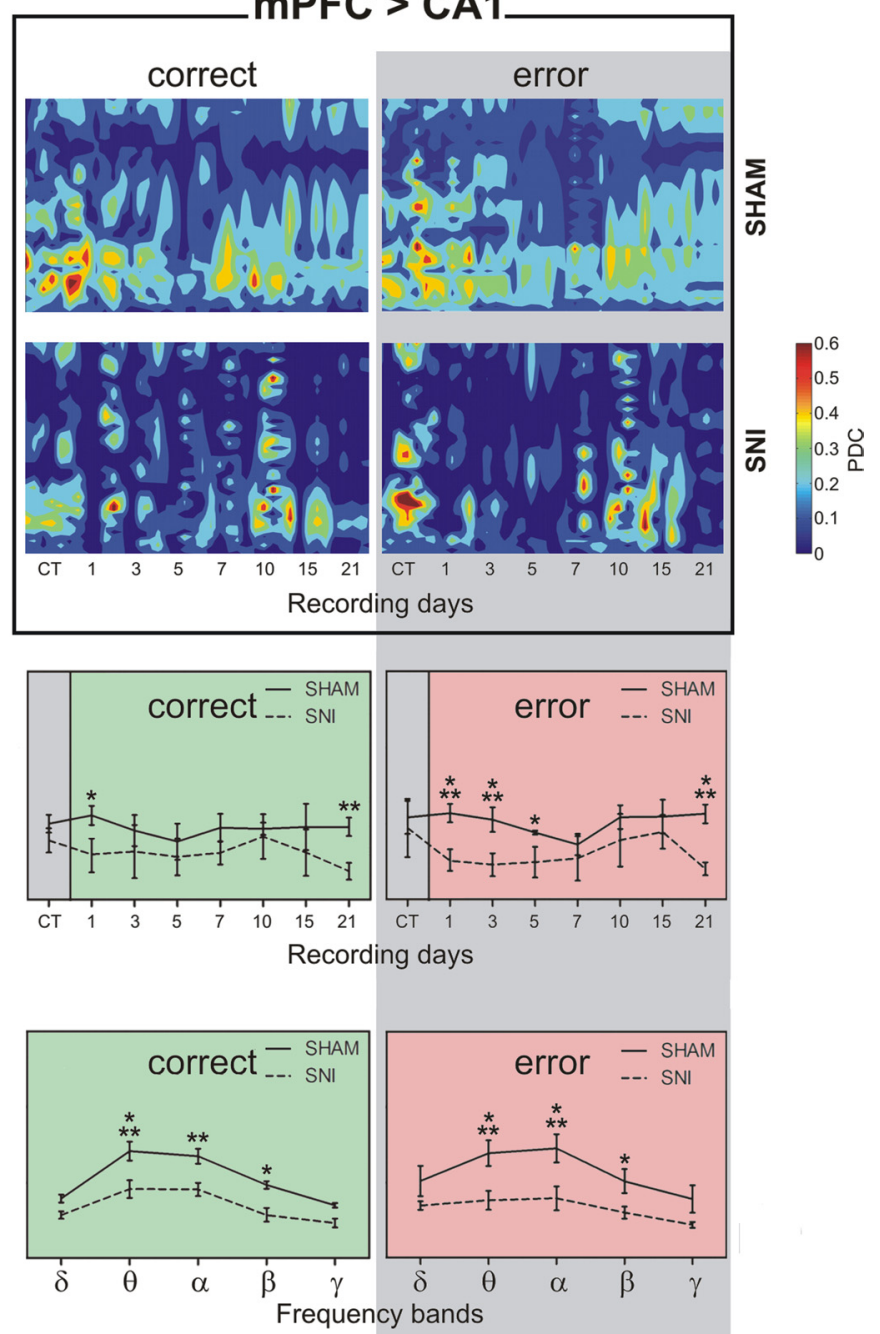

Figure 10. Patterns of information flow activity for correct and error alternations. Different patterns of PDC activity were observed across correct and error alternations. $\boldsymbol{A}$, Note, for example, a strong theta band activity during correct alternations for the CA1 $\rightarrow \mathrm{mPFC}$ direction, which is altered after peripheral nerve lesioning. $\boldsymbol{B}$, In terms of recording sessions, the averaged PDC activity (in the $1-50 \mathrm{~Hz}$ frequency range) indicates that significant differences were found between experimental groups. $\boldsymbol{C}$, In terms of frequency bands, the PDC activity showed that less information was processed in both directions of the circuit across theta, alpha, and beta frequency bands for correct and error alternations after nerve lesioning. Frequency band analysis corresponds to the averaged PDC activity across postsurgery sham/SNI recording sessions. Frequency bands: delta $(\delta ; 1-4 \mathrm{~Hz})$, theta $(\theta ; 4-9 \mathrm{~Hz})$, alpha $(\alpha ; 9-15 \mathrm{~Hz})$, beta $(\beta ; 15-30 \mathrm{~Hz})$, and slow-gamma $(\gamma ; 30-50 \mathrm{~Hz})$. Values are presented as the mean \pm SEM. SNI group, $n=5$; sham group, $n=5$. Comparisons between experimental groups are based on two-factor repeated-measures ANOVA, followed by Bonferroni post hoc test. ${ }^{*} p<0.05,{ }^{* *} p<0.01,{ }^{* * *} p<0.001$.

contrast with another study showing that an increased theta entrainment correlated with enhanced memory performance (Hyman et al., 2010). However, the biological role of frontohippocampal theta entrainment is still a disputed matter (Hyman et al., 2011). For example, similar increases in theta entrainment have been described in anxious animals (Adhikari et al., 2010), which is particularly relevant for our results since neuropathic animals have increased anxiety (Bravo et al., 2012).

Although the LFPs activity did not present changes in power spectrum, both recorded brain regions shared a prominent theta power band, as has previously been described in other research reports (Buzsáki, 2002; Lörincz et al., 2007). An intensification of theta-range coherence across frontal and hippocampal regions has been described during the execution of working memory tasks using human EEG recordings (Tesche and Karhu, 2000; Onton et al., 2005). More recently, an elevation of mPFC gammarange power has been associated with the maintenance of working memory processes (Izaki and Akema, 2008; Klimesch et al.,
2008; Sirota et al., 2008; Colgin et al., 2009). In our study, the mPFC-dCA1 LFP coherence changed across experimental groups and recording sessions, while no significant pain-related differences were found across frequency bands. However, it must be noted that, in our case, all coherence analysis compared long periods of activity, which necessarily results in smaller differences.

Previous studies have demonstrated that neural activity in the $\mathrm{mPFC}$ is synchronized with theta frequency oscillations in the dorsal hippocampus (Hyman et al., 2005; Jones and Wilson, 2005b), despite the fact that these two regions are indirectly connected (Hoover and Vertes, 2007). In contrast, the ventral hippocampus and the $\mathrm{MPFC}$ are directly connected (Verwer et al., 1997), and in this case it was found that over a broad range of frequencies the mPFC is highly coherent with the ventral hippocampus but not with the dorsal hippocampus, and that this coherency was modulated by anxiety (Adhikari et al., 2010). However, it must be noted that coherent activity between two 
signals suggests synchronization but does not disambiguate whether the synchrony is due to correlated fluctuations in power (which relates to oscillation amplitude) or is due to a consistent phase relationship between the two signals (which relates to oscillation timing).

To further study the relationship of activity between hippocampus and the mPFC, we calculated the information flow dynamics in the circuit using partial directed coherence analysis. In fact, the most significant finding in the present study is that the onset of neuropathic pain causes a decrease in mPFC-hippocampus flow of information as measured by PDC (Sameshima and Baccalá, 1999; Baccalá and Sameshima, 2001); this decrease was observed in both directions of the circuit across reward and choice navigation zones, despite the lack of alteration in the $\mathrm{mP}$ FC-hippocampus power spectrum.

Several authors have suggested that pain is one of the leading factors that can induce disruptions of spontaneous oscillations at different cortico-subcortical circuits (Corbetta and Shulman, 2002; Mouraux et al., 2003; Ohara et al., 2004), including the inhibition or potentiation of oscillatory rhythms (Backonja et al., 1991; Chang et al., 2002; Ploner et al., 2004). Oscillatory interactions across the different brain circuits reflect a global functional state of the system and are used as an alert mechanism to find normal functional state perturbations (Ploner et al., 2006). In what concerns processing of nociceptive information, an example of these perturbations has recently been reported in the rat thalamocortical circuit using the same chronic pain model applied to this study (Cardoso-Cruz et al., 2011b). Additionally, it has recently been suggested that the transition from acute to chronic pain in humans was correlated with an initially greater connectivity level between nucleus accumbens and mPFC (Baliki et al., 2012).

It should be noted that the reduction in task performance could result from pain-induced motor impairment of the animals; indeed, both groups experienced a reduction of the navigation velocity following the sham or SNI surgery, which resolved to presurgery levels of movement after 1 week. However, we believe that this motor impairment is not the leading cause of the performance difference between groups because it similarly affected both sham and nerve-lesioned animals and because the video-tracking measurement of instantaneous speed showed similar running speeds in both groups (Fig. 1F). Moreover, the observed difference between groups in alternation duration is caused by the longer trajectories and frequent pauses at transition points done by the nerve-lesioned animals; this increase in the average time of navigation also leads to longer delays between alternations, which results in more challenging tasks (Lee and Kesner, 2003).

Another important issue is the validation of the hippocampal normal function dependency, because our task protocol contains considerable training. Packard (2009) found that on tasks similar to the path alternation task we used in this study, the behavior progresses from a strict hippocampus-dependent to a hippocampus-independent striatum based habit. Thus, the changes we observed in hippocampal activity could have no real effect on spatial working memory performance. To test whether our task protocol indeed requires hippocampal functioning, we tested both hippocampus-lesioned animals and intact animals in a hippocampus-dependent T-maze. The hippocampus-lesioned animals revealed a significant decrease of their performance on the task, showing that hippocampal function dependency remains unchanged across the training schedule even with task overtraining, suggesting that a causal link between electrophysiological recordings and behavior is still valid.

In summary, our data suggest that peripheral nerve injury (SNI) causes impairment in spatial working memory performance. In terms of the temporal structure of spiking and LFP activity in the $\mathrm{mPFC}-\mathrm{dCA} 1$ circuit, both populations of neurons increased their spiking phase precision with respect to hippocampal theta rhythm after nerve lesioning. In addition, our data showed a clear reduction of the amount of information shared by this circuit, which occurs at different frequency bands depending on whether the rat performs a correct or error alternation. These changes are probably caused by adaptive mechanisms that occur during the onset of a painful condition (Saab, 2012), which may disturb the mnemonic processes that rely on the integration and consolidation of spatial working memory.

\section{References}

Adhikari A, Topiwala MA, Gordon JA (2010) Synchronized activity between the ventral hippocampus and the medial prefrontal cortex during anxiety. Neuron 65:257-269. CrossRef Medline

Aguiar P, Mendonça L, Galhardo V (2007) OpenControl: a free opensource software for video tracking and automated control of behavioral mazes. J Neurosci Methods 166:66-72. CrossRef Medline

Al-Amin H, Sarkis R, Atweh S, Jabbur S, Saadé N (2011) Chronic dizocilpine or apomorphine and development of neuropathy in two animal models II: effects on brain cytokines and neurotrophins. Exp Neurol 228: 30-40. CrossRef Medline

Apkarian AV, Hashmi JA, Baliki MN (2011) Pain and the brain: specificity and plasticity of the brain in clinical chronic pain. Pain 152 [3 Suppl]: S49-S64. CrossRef

Baccalá LA, Sameshima K (2001) Partial directed coherence: a new concept in neural structure determination. Biol Cybern 84:463-474. CrossRef Medline

Backonja M, Howland EW, Wang J, Smith J, Salinsky M, Cleeland CS (1991) Tonic changes in alpha power during immersion of the hand in cold water. Electroencephalogr Clin Neurophysiol 79:192-203. CrossRef Medline

Baeg EH, Kim YB, Huh K, Mook-Jung I, Kim HT, Jung MW (2003) Dynamics of population code for working memory in the prefrontal cortex. Neuron 40:177-188. CrossRef Medline

Baliki MN, Petre B, Torbey S, Herrmann KM, Huang L, Schnitzer TJ, Fields HL, Apkarian AV (2012) Corticostriatal functional connectivity predicts transition to chronic back pain. Nat Neurosci 15:1117-1119. CrossRef Medline

Boyette-Davis JA, Thompson CD, Fuchs PN (2008) Alterations in attentional mechanisms in response to acute inflammatory pain and morphine administration. Neuroscience 151:558-563. CrossRef Medline

Bravo L, Mico JA, Rey-Brea R, Pérez-Nievas B, Leza JC, Berrocoso E (2012) Depressive-like states heighten the aversion to painful stimuli in a rat model of comorbid chronic pain and depression. Anesthesiology 117: 613-625. CrossRef Medline

Buhle J, Wager TD (2010) Performance-dependent inhibition of pain by an executive working memory task. Pain 149:19-26. CrossRef Medline

Buzsáki G (2002) Theta oscillations in the hippocampus. Neuron 33:325340. CrossRef Medline

Cain CK, Francis JM, Plone MA, Emerich DF, Lindner MD (1997) Painrelated disability and effects of chronic morphine in the adjuvant-induced arthritis model of chronic pain. Physiol Behav 62:199-205. CrossRef Medline

Cardoso-Cruz H, Lima D, Galhardo V (2011a) Instability of spatial encoding by CA1 hippocampal place cells after peripheral nerve injury. Eur J Neurosci 33:2255-2264. CrossRef Medline

Cardoso-Cruz H, Sameshima K, Lima D, Galhardo V (2011b) Dynamics of circadian thalamocortical flow of information during a peripheral neuropathic pain condition. Front Integr Neurosci 5:43. CrossRef Medline

Chang PF, Arendt-Nielsen L, Chen AC (2002) Differential cerebral responses to aversive auditory arousal versus muscle pain: specific EEG patterns are associated with human pain processing. Exp Brain Res 147: 387-393. CrossRef Medline

Chaplan SR, Bach FW, Pogrel JW, Chung JM, Yaksh TL (1994) Quanti- 
tative assessment of tactile allodynia in the rat paw. J Neurosci Methods 53:55-63. CrossRef Medline

Colgin LL, Denninger T, Fyhn M, Hafting T, Bonnevie T, Jensen O, Moser MB, Moser EI (2009) Frequency of gamma oscillations routes flow of information in the hippocampus. Nature 462:353-357. CrossRef Medline

Corbetta M, Shulman GL (2002) Control of goal-directed and stimulusdriven attention in the brain. Nat Rev Neurosci 3:201-215. Medline

Decosterd I, Woolf CJ (2000) Spared nerve injury: an animal model of persistent peripheral neuropathic pain. Pain 87:149-158. CrossRef Medline

del Rey A, Yau HJ, Randolf A, Centeno MV, Wildmann J, Martina M, Besedovsky HO, Apkarian AV (2011) Chronic neuropathic pain-like behavior correlates with IL- $1 \beta$ expression and disrupts cytokine interactions in the hippocampus. Pain 152:2827-2835. CrossRef Medline

Devoize L, Alvarez P, Monconduit L, Dallel R (2011) Representation of dynamic mechanical allodynia in the ventral medial prefrontal cortex of trigeminal neuropathic rats. Eur J Pain 15:676-682. CrossRef Medline

Ducreux D, Attal N, Parker F, Bouhassira D (2006) Mechanisms of central neuropathic pain: a combined psychophysical and fMRI study in syringomyelia. Brain 129:963-976. CrossRef Medline

Floresco SB, Seamans JK, Phillips AG (1997) Selective roles for hippocampal, prefrontal cortical, and ventral striatal circuits in radial-arm maze tasks with or without a delay. J Neurosci 17:1880-1890. Medline

Fox SE, Ranck JB Jr (1981) Electrophysiological characteristics of hippocampal complex-spike cells and theta cells. Exp Brain Res 41:399-410. Medline

Frank Y, Seiden J, Napolitano B (1996) Visual event related potentials and reaction time in normal adults, normal children, and children with attention deficit hyperactivity disorder: differences in short-term memory processing. Int J Neurosci 88:109-124. CrossRef Medline

Fuster JM (1997) Network memory. Trends Neurosci 20:451-459. CrossRef Medline

Gaskin S, Tardif M, Mumby DG (2009a) Patterns of retrograde amnesia for recent and remote incidental spatial learning in rats. Hippocampus 19: 1212-1221. CrossRef Medline

Gaskin S, Gamliel A, Tardif M, Cole E, Mumby DG (2009b) Incidental (unreinforced) and reinforced spatial learning in rats with ventral and dorsal lesions of the hippocampus. Behav Brain Res 202:64-70. CrossRef Medline

Green MF (2006) Cognitive impairment and functional outcome in schizophrenia and bipolar disorder. J Clin Psychiatry 67:e12. CrossRef Medline

Hoover WB, Vertes RP (2007) Anatomical analysis of afferent projections to the medial prefrontal cortex in the rat. Brain Struct Funct 212:149-179. CrossRef Medline

Hu Y, Yang J, Hu Y, Wang Y, Li W (2010) Amitriptyline rather than lornoxicam ameliorates neuropathic pain-induced deficits in abilities of spatial learning and memory. Eur J Anaesthesiol 27:162-168. CrossRef Medline

Hyman JM, Zilli EA, Paley AM, Hasselmo ME (2005) Medial prefrontal cortex cells show dynamic modulation with the hippocampal theta rhythm dependent on behavior. Hippocampus 15:739-749. CrossRef Medline

Hyman JM, Zilli EA, Paley AM, Hasselmo ME (2010) Working memory performance correlates with prefrontal-hippocampal theta interactions but not with prefrontal neuron firing rates. Front Integr Neurosci 4:2. CrossRef Medline

Hyman JM, Hasselmo ME, Seamans JK (2011) What is the functional relevance of prefrontal cortex entrainment to hippocampal theta rhythms? Front Neurosci 5:24. CrossRef Medline

Izaki Y, Akema T (2008) Gamma-band power elevation of prefrontal local field potential after posterior dorsal hippocampus-prefrontal long-term potentiation induction in anesthetized rats. Exp Brain Res 184:249-253. CrossRef Medline

Ji D, Wilson MA (2007) Coordinated memory replay in the visual cortex and hippocampus during sleep. Nat Neurosci 10:100-107. CrossRef Medline

Ji G, Sun H, Fu Y, Li Z, Pais-Vieira M, Galhardo V, Neugebauer V (2010) Cognitive impairment in pain through amygdala-driven prefrontal cortical deactivation. J Neurosci 30:5451-5464. CrossRef Medline

Jones MW, Wilson MA (2005a) Phase precession of medial prefrontal cortical activity relative to the hippocampal theta rhythm. Hippocampus 15:867-873. CrossRef Medline

Jones MW, Wilson MA (2005b) Theta rhythms coordinate hippocampal- prefrontal interactions in a spatial memory task. PLoS Biol 3:e402. CrossRef Medline

Jung MW, Qin Y, McNaughton BL, Barnes CA (1998) Firing characteristics of deep layer neurons in prefrontal cortex in rats performing spatial working memory tasks. Cereb Cortex 8:437-450. CrossRef Medline

Kim SH, Kim SH, Kim SK, Nam EJ, Han SW, Lee SJ (2012) Spatial versus verbal memory impairments in patients with fibromyalgia. Rheumatol Int 32:1135-1142. CrossRef Medline

Klimesch W, Freunberger R, Sauseng P, Gruber W (2008) A short review of slow phase synchronization and memory: evidence for control processes in different memory systems? Brain Res 1235:31-44. CrossRef Medline

Kodama D, Ono H, Tanabe M (2007) Altered hippocampal long-term potentiation after peripheral nerve injury in mice. Eur J Pharmacol 574:127132. CrossRef Medline

Kyd RJ, Bilkey DK (2003) Prefrontal cortex lesions modify the spatial properties of hippocampal place cells. Cereb Cortex 13:444-451. CrossRef Medline

Lee I, Kesner RP (2003) Time-dependent relationship between the dorsal hippocampus and the prefrontal cortex in spatial memory. J Neurosci 23:1517-1523. Medline

Legrain V, Crombez G, Mouraux A (2011) Controlling attention to nociceptive stimuli with working memory. PLoS One 6:e20926. CrossRef Medline

Leite-Almeida H, Almeida-Torres L, Mesquita AR, Pertovaara A, Sousa N, Cerqueira JJ, Almeida A (2009) The impact of age on emotional and cognitive behaviours triggered by experimental neuropathy in rats. Pain 144:57-65. CrossRef Medline

Lindner MD, Plone MA, Francis JM, Cain CK (1999) Chronic morphine reduces pain-related disability in a rodent model of chronic, inflammatory pain. Exp Clin Psychopharmacol 7:187-197. CrossRef Medline

Ling J, Campbell C, Heffernan TM, Greenough CG (2007) Short-term prospective memory deficits in chronic back pain patients. Psychosom Med 69:144-148. CrossRef Medline

Lisman J (2010) Working memory: the importance of theta and gamma oscillations. Curr Biol 20:490-492. CrossRef

Lörincz M, Oláh M, Baracskay P, Szilágyi N, Juhász G (2007) Propagation of spike and wave activity to the medial prefrontal cortex and dorsal raphe nucleus of WAG/Rij rats. Physiol Behav 90:318-324. CrossRef Medline

Luerding R, Weigand T, Bogdahn U, Schmidt-Wilcke T (2008) Working memory performance is correlated with local brain morphology in the medial frontal and anterior cingulate cortex in fibromyalgia patients: structural correlates of pain-cognition interaction. Brain 131:3222-3231. CrossRef Medline

McDowell S, Whyte J, D’Esposito M (1997) Working memory impairments in traumatic brain injury: evidence from a dual-task paradigm. Neuropsychologia 35:1341-1353. CrossRef Medline

Metz AE, Yau HJ, Centeno MV, Apkarian AV, Martina M (2009) Morphological and functional reorganization of rat medial prefrontal cortex in neuropathic pain. Proc Natl Acad Sci U S A 106:2423-2428. CrossRef Medline

Millecamps M, Etienne M, Jourdan D, Eschalier A, Ardid D (2004) Decrease in non-selective, non-sustained attention induced by a chronic visceral inflammatory state as a new pain evaluation in rats. Pain 109:214-224. CrossRef Medline

Miller EK, Erickson CA, Desimone R (1996) Neural mechanisms of visual working memory in prefrontal cortex of the macaque. J Neurosci 16: 5154-5167. Medline

Moriarty O, McGuire BE, Finn DP (2011) The effect of pain on cognitive function: a review of clinical and preclinical research. Prog Neurobiol 93:385-404. CrossRef Medline

Mouraux A, Guérit JM, Plaghki L (2003) Non-phase locked electroencephalogram (EEG) responses to $\mathrm{CO} 2$ laser skin stimulations may reflect central interactions between a partial partial differential- and C-fibre afferent volleys. Clin Neurophysiol 114:710-722. CrossRef Medline

Mutso AA, Radzicki D, Baliki MN, Huang L, Banisadr G, Centeno MV, Radulovic J, Martina M, Miller RJ, Apkarian AV (2012) Abnormalities in hippocampal functioning with persistent pain. J Neurosci 32:5747-5756. CrossRef Medline

Ohara S, Crone NE, Weiss N, Lenz FA (2004) Attention to a painful cutaneous laser stimulus modulates electrocorticographic event-related desynchronization in humans. Clin Neurophysiol 115:1641-1652. CrossRef Medline 
Onton J, Delorme A, Makeig S (2005) Frontal midline EEG dynamics during working memory. Neuroimage 27:341-356. CrossRef Medline

Packard MG (2009) Anxiety, cognition, and habit: a multiple memory systems perspective. Brain Res 1293:121-128. CrossRef Medline

Pais-Vieira M, Mendes-Pinto MM, Lima D, Galhardo V (2009a) Cognitive impairment of prefrontal-dependent decision-making in rats after the onset of chronic pain. Neuroscience 161:671-679. CrossRef Medline

Pais-Vieira M, Lima D, Galhardo V (2009b) Sustained attention deficits in rats with chronic inflammatory pain. Neurosci Lett 463:98-102. CrossRef Medline

Pais-Vieira M, Aguiar P, Lima D, Galhardo V (2012) Inflammatory pain disrupts the orbitofrontal neuronal activity and risk-assessment performance in a rodent decision-making task. Pain 153:1625-1635. CrossRef Medline

Paxinos G, Watson C (1998) The rat brain in stereotaxic coordinates. San Diego: Academic.

Pesaran B, Pezaris JS, Sahani M, Mitra PP, Andersen RA (2002) Temporal structure in neuronal activity during working memory in macaque parietal cortex. Nat Neurosci 5:805-811. CrossRef Medline

Peyron R, García-Larrea L, Grégoire MC, Convers P, Lavenne F, Veyre L, Froment JC, Mauguière F, Michel D, Laurent B (1998) Allodynia after lateral-medullary (Wallenberg) infarct. A PET study. Brain 121:345-356. CrossRef Medline

Ploghaus A, Tracey I, Clare S, Gati JS, Rawlins JN, Matthews PM (2000) Learning about pain: the neural substrate of the prediction error for aversive events. Proc Natl Acad Sci U S A 97:9281-9286. CrossRef Medline

Ploner M, Pollok B, Schnitzler A (2004) Pain facilitates tactile processing in human somatosensory cortices. J Neurophysiol 92:1825-1829. CrossRef Medline

Ploner M, Gross J, Timmermann L, Pollok B, Schnitzler A (2006) Pain suppresses spontaneous brain rhythms. Cereb Cortex 16:537-540. CrossRef Medline

Rainer G, Rao SC, Miller EK (1999) Prospective coding for objects in primate prefrontal cortex. J Neurosci 19:5493-5505. Medline

Ren WJ, Liu Y, Zhou LJ, Li W, Zhong Y, Pang RP, Xin WJ, Wei XH, Wang J, Zhu HQ, Wu CY, Qin ZH, Liu G, Liu XG (2011) Peripheral nerve injury leads to working memory deficits and dysfunction of the hippocampus by upregulation of TNF- $\alpha$ in rodents. Neuropsychopharmacology 36:979992. CrossRef Medline

Ruan M, Young CK, McNaughton N (2011) Minimal driving of hippocampal theta by the supramammillary nucleus during water maze learning. Hippocampus 21:1074-1081. CrossRef Medline

Saab CY (2012) Pain-related changes in the brain: diagnostic and therapeutic potentials. Trends Neurosci 35:629-637. CrossRef Medline

Sameshima K, Baccalá LA (1999) Using partial directed coherence to de- scribe neuronal ensemble interactions. J Neurosci Methods 94:93-103. CrossRef Medline

Schon K, Atri A, Hasselmo ME, Tricarico MD, LoPresti ML, Stern CE (2005) Scopolamine reduces persistent activity related to long-term encoding in the parahippocampal gyrus during delayed matching in humans. J Neurosci 25:9112-9123. CrossRef Medline

Schweinhardt P, Glynn C, Brooks J, McQuay H, Jack T, Chessell I, Bountra C, Tracey I (2006) An fMRI study of cerebral processing of brush-evoked allodynia in neuropathic pain patients. Neuroimage 32:256-265. CrossRef Medline

Seifert F, Bschorer K, De Col R, Filitz J, Peltz E, Koppert W, Maihöfner C (2009) Medial prefrontal cortex activity is predictive for hyperalgesia and pharmacological antihyperalgesia. J Neurosci 29:6167-6175. CrossRef Medline

Siapas AG, Lubenov EV, Wilson MA (2005) Prefrontal phase locking to hippocampal theta oscillations. Neuron 46:141-151. CrossRef Medline

Sirota A, Montgomery S, Fujisawa S, Isomura Y, Zugaro M, Buzsáki G (2008) Entrainment of neocortical neurons and gamma oscillations by the hippocampal theta rhythm. Neuron 60:683-697. CrossRef Medline

Skaggs WE, McNaughton BL, Wilson MA, Barnes CA (1996) Theta phase precession in hippocampal neuronal populations and the compression of temporal sequences. Hippocampus 6:149-172. CrossRef Medline

Stern CE, Sherman SJ, Kirchhoff BA, Hasselmo ME (2001) Medial temporal and prefrontal contributions to working memory tasks with novel and familiar stimuli. Hippocampus 11:337-346. CrossRef Medline

Suzuki T, Amata M, Sakaue G, Nishimura S, Inoue T, Shibata M, Mashimo T (2007) Experimental neuropathy in mice is associated with delayed behavioral changes related to anxiety and depression. Anesth Analg 104: 1570-1577. CrossRef Medline

Tesche CD, Karhu J (2000) Theta oscillations index human hippocampal activation during a working memory task. Proc Natl Acad Sci U S A 97: 919-924. CrossRef Medline

Vallar G, Papagno C (1993) Preserved vocabulary acquisition in Down's syndrome: the role of phonological short-term memory. Cortex 29:467483. Medline

Verwer RW, Meijer RJ, Van Uum HF, Witter MP (1997) Collateral projections from the rat hippocampal formation to the lateral and medial prefrontal cortex. Hippocampus 7:397-402. CrossRef Medline

Wang GW, Cai JX (2006) Disconnection of the hippocampal-prefrontal cortical circuits impairs spatial working memory performance in rats. Behav Brain Res 175:329-336. CrossRef Medline

Yoon T, Okada J, Jung MW, Kim JJ (2008) Prefrontal cortex and hippocampus subserve different components of working memory in rats. Learn Mem 15:97-105. CrossRef Medline

Zimmermann M (1983) Ethical guidelines for investigations of experimental pain in conscious animals. Pain 16:109-110. CrossRef Medline 